\title{
Fractional-order theory of heat transport in rigid bodies
}

\author{
Massimiliano Zingales*
}

\begin{abstract}
Dipartimento di Ingegneria Civile, Ambientale, Aerospaziale, dei Materiali, Viale delle Scienze Ed.8, 90128 Palermo, Italy Bio-Nanomechanics in Medicine (BNM2) Laboratory, Mediterranean Center of Human Health and Advanced Biotechnologies (MED-CHHAB), Viale delle Scienze Ed.18, 90128 Palermo, Italy
\end{abstract}

\section{A R T I C L E I N F O}

\section{Article history:}

Received 7 October 2013

Received in revised form 16 April 2014

Accepted 17 April 2014

Available online 30 April 2014

\section{Keywords:}

Fractional derivatives

Non-local thermodynamics

Generalized entropy

Temperature field

Non-local temperature gradients

\begin{abstract}
A B S T R A C T
The non-local model of heat transfer, used to describe the deviations of the temperature field from the well-known prediction of Fourier/Cattaneo models experienced in complex media, is framed in the context of fractional-order calculus. It has been assumed (Borino et al., 2011 [53], Mongioví and Zingales, 2013 [54]) that thermal energy transport is due to two phenomena: (i) A short-range heat flux ruled by a local transport equation; (ii) A long-range thermal energy transfer proportional to a distance-decaying function, to the relative temperature and to the product of the interacting masses. The distance-decaying function is assumed in the functional class of the power-law decay of the distance yielding a novel temperature equation in terms of $\alpha$-order Marchaud fractional-order derivative $(0 \leqslant \alpha \leqslant 1)$. Thermodynamical consistency of the model is provided in the context of Clausius-Plank inequality. The effects induced by the boundary conditions on the temperature field are investigated for diffusive as well as ballistic local heat flux. Deviations of the temperature field from the linear distributions in the neighborhood of the thermostated zones of small-scale conductors are qualitatively predicted by the used fractional-order heat transport model, as shown by means of molecular dynamics simulations.
\end{abstract}

(c) 2014 Elsevier B.V. All rights reserved.

\section{Introduction}

The need for non-local thermodynamics in physical sciences and engineering may be traced back to the mid of the last century in the attempt to capture the experimental effects unpredicted by Fourier diffusion theory. Indeed several experimental observations of temperature field at metal interfaces as well as of the changes in conductivity parameters in the neighborhood of thermostated regions (Kapitsa phonon-scattering) shows a localization of temperature gradients close to the borders [1].

Similar phenomena have been observed with molecular dynamics (MD) simulations of heat transfer in nanowires showing that the presence of thermostated regions involves a phonon-phonon scattering that modifies the conductivity property of the materials $[2,3]$.

Such studies have been further developed toward the use of advanced mathematical tools as the fractional-order calculus [4] to capture memory [5,6] as well as non-local effects [7-9]. Indeed fractional (real) order integro-differential operators have been introduced more and more often in several contexts of physics and engineering for their capability to interpolate among the well-known integer-order operators of classical differential calculus [10]. In this regard some applications may be

* Address: Dipartimento di Ingegneria Civile, Ambientale, Aerospaziale, dei Materiali, Viale delle Scienze Ed.8, 90128 Palermo, Italy. Tel.: +39 091 23896763.

E-mail address: massimiliano.zingales@unipa.it 
found in the study of temporal and spatial evolution of complex systems close to critical points [11-14] or in stochastic setting [15-17]. Fractional-order differential calculus is widely used, also, to model the mechanical behavior of polymers, gels, foams and glassy materials [18-22] but also to model the rheology of soft matter and biological tissues [23-25]. States and free energies for non-linear geometries [26-30] in terms of fractional-order derivatives may also be formulated.

The long-tails of fractional operators have been used to formulate non-local stress-strain constitutive equations that are a particularized version of the integral model of non-local elasticity [31,32]. The same feature has been also used by the author and its research team to derive a mechanically-based fractional-order non-local elasticity in statics [33-36] and wave propagation contexts [37,38] (see e.g. [39] for a complete review).

The presence of spatial non-local effects, observed in heat transport framework, has been introduced by means of integral models involving, beside the local gradient of the temperature field, an integral convolution among the temperature gradient and a real-order attenuation function [40-42]. The non-local formulation, originally proposed by Eringen and his co-workers, has been used, recently, to model thermoelastic coupling in microelectromechanical resonators (MEMRS) [43,44]. Some generalization of this theory may be useful to the analysis of small-scale systems accounting for second-sound effects [45-47] modeled with a first-order time derivative of the heat flux [48-50] and introducing a generalized entropy [51,52].

Very recently a non-local model of thermal energy transport has been proposed with a physical picture of heat transfer in 1D setting. It has been assumed that the non-local residual in the balance equation is due to a volume integral over the body domain of the elementary long-range heat transport among adjacent and non-adjacent locations of the body [53]. The longrange thermal energy contribution is modeled as two point function $\mathcal{P}^{(n l)}(\mathbf{x}, \mathbf{y}, t)$ that depends on: (i) A decaying function decreasing with the distance of the interacting elements; (ii) The relative temperatures among locations and (iii) The product of the masses at locations $\mathbf{x}$ and $\mathbf{y}$ [54].

In this paper it is shown that, assuming the decaying function in the functional class of power-laws of the distance, the balance principle involves fractional-order non-local residuals. The correspondent temperature equation is obtained in terms of Marchaud-type fractional derivatives in unbounded domains. A different scenario appears as the thermal energy exchange in bounded domains is considered since only the integral contributions to the Marchaud fractional derivatives defined on bounded regions appear. It follows that the divergent algebraic contributions at the borders are not included in the formulation allowing for the position of non-homogeneous Dirichlet boundary conditions straightforwardly. Moreover the Neumann boundary conditions associated to the fractional-order temperature equation involve, only, the gradient of the temperature field as in well-known local heat transport theories.

The effects induced by the non-homogeneous boundary condition is further investigated, in this paper, either for diffusive and ballistic/diffusive thermal energy exchange. The numerical results reported in the analyses describe the temperature field in 1D rigid conductors showing that the proposed model of fractional-order thermal energy exchange may capture the non-uniform temperature distribution observed in Kapitsa experiments as well as in molecular dynamics simulations.

\section{The fractional model of thermal energy exchange in rigid bodies: the second law of thermodynamics}

In this section the fractional-order model of thermal energy exchange is derived for a diffusive heat transport. In the first part of the section the balance equation as well as the second law of thermodynamics will be shortly recalled. The second part of the section is dedicated to a numerical simulation of the temperature field in a 1D rigid conductor in presence of longrange thermal energy transport. The effects of the differentiation order on the temperature field in bounded conductors have been addressed with a numerical simulation code.

The main idea beyond the proposed model of non-local thermodynamics relies on the assumption that the energy balance at location $\mathbf{x} \in \mathbb{R}^{3}$ of a rigid body, encapsulated in a subset $V \subset \mathbb{R}^{3}$ with boundary surface $S=\partial V$, involves the following contributions:

1. The thermal energy flux among adjacent locations, that it is related to the divergence $\nabla \cdot \mathbf{q}(\mathbf{x}, t)$ of the heat flux density vector $\mathbf{q}(\mathbf{x}, t)$.

2. A non-local energy transfer, due to the contribution of the elements $\mathbf{y} \in \mathbb{R}^{3}$ of the body, that it is assumed proportional to the mass densities of the interacting elements at the locations $\mathbf{x}$ and $\mathbf{y}$ as

$$
\mathcal{P}^{(n l)}(\mathbf{x}, \mathbf{y} ; t)=\chi^{(n l)}(\mathbf{x}, \mathbf{y} ; t) \rho(\mathbf{x}) \rho(\mathbf{y}) d V_{\mathbf{x}} d V_{\mathbf{y}}
$$

where $\chi^{(n l)}(\mathbf{x}, \mathbf{y} ; t) \rho(\mathbf{y}) d V_{\mathbf{y}}$ is the long-range specific energy per unit time transferred at locations $\mathbf{x}$ by the element at the location $\mathbf{y}$ and $\rho$ is the mass density that is time-independent. Under some restriction of the functional dependence of the longrange specific energy $\mathcal{P}^{(n l)}(\mathbf{x}, \mathbf{y} ; t)$, a Marchaud-type, fractional-order, non-local model of thermal energy transport is obtained in unbounded domains. In bounded domains, instead, only integral parts of fractional-order operators are involved.

This latter consideration yields two key features of the fractional model of long-range heat transport: $(i)$ The Non-Homogeneous Dirichlet boundary conditions of the temperature field along the boundary $S_{d}$, namely $T(\mathbf{x}, t)=\bar{T}(\mathbf{x}, t)$ with $\mathbf{x} \in S_{d}$ may be easily accounted for since the divergent algebraic contribution to the Marchaud fractional derivatives do not appear; (ii) The Neumann boundary conditions on the free surface $S_{n}$ involves, only, the local contribution to the heat transfer in terms of gradients of the temperature field $\nabla \cdot T(\mathbf{x}, t)$ with $\mathbf{x} \in S_{n}$ since the overall residual reads: 


$$
\int_{V_{\mathbf{x}}} \int_{V_{\mathbf{y}}} \chi^{(n l)}(\mathbf{x}, \mathbf{y} ; t) \rho(\mathbf{y}) \rho(\mathbf{x}) d V_{\mathbf{y}} d V_{\mathbf{x}}=0
$$

for any specific class of the specific long-range heat transport $\chi^{(n l)}(\mathbf{x}, \mathbf{y} ; t) \rho(\mathbf{y}) d V_{\mathbf{y}}$ [54].

\subsection{The fractional-order theory of Classical Irreversible Thermodynamics (CIT)}

Let us consider an isotropic solid body with $u:=u(\mathbf{x} ; t)$ the specific internal energy at location $\mathbf{x}$ and time $t$ and let us assume that $\varrho=\varrho(\mathbf{x})$ is the time-independent body density at location $\mathbf{x}$ as for closed thermodynamical system. The absolute temperature of the body is denoted as $T(\mathbf{x}, t)$, and $C_{V}=\left(\frac{\partial u}{\partial T}\right)_{T_{0}}$ is the volume specific heat at room temperature $T_{0}$ assumed constant in the analysis.

In the context of non-local thermodynamics we assume that, at location $\mathbf{x}=\left(x_{1}, x_{2}, x_{3}\right)$ of the body, the internal energy of the body $\varrho(\mathbf{x}) u(\mathbf{x}, t)$ is composed by two contributes as:

$$
\frac{\partial(\varrho(\mathbf{x}) u)}{\partial t}=\frac{\partial\left(\varrho(\mathbf{x}) u_{l}\right)}{\partial t}+\frac{\partial\left(\varrho(\mathbf{x}) u_{n l}\right)}{\partial t}
$$

where we denoted $\varrho(\mathbf{x}) u_{l}(\mathbf{x}, t)$ and $\varrho(\mathbf{x}) u_{n l}(\mathbf{x}, t)$ the local and the long-range overall contribution to the internal energy at location $\mathbf{x}$, respectively. In this regard the rate of change of the internal energy function in the balance equation ( 3 ) for a closed thermodynamical system reads:

$$
\frac{\partial u}{\partial t}=\frac{\partial u_{l}}{\partial t}+\frac{\partial u_{n l}}{\partial t}=\dot{w}(\mathbf{x}, t)+\dot{h}(\mathbf{x}, t)=\dot{h}_{l}(\mathbf{x}, t)+\dot{h}_{n l}(\mathbf{x}, t)
$$

where $\dot{w}=0$ since rigid conductors are considered and $\dot{h}$ is the rate of change of the specific thermal energy that is composed by a local $\dot{h}_{l}(\mathbf{x}, t)$ and a long-range $\dot{h}_{n l}(\mathbf{x}, t)$ term, respectively. The latter equality in Eq. (4) is the local version of first principle of thermodynamics in presence of long-range thermal energy transport yielding that the rate of change of the specific internal energy $\dot{u}(\mathbf{x}, t)$, equates the rate of change of the thermal energy $\dot{h}(\mathbf{x}, t)$ in any subdomain $d V_{\mathbf{x}}$ of the conductor.

The proposed model of thermal energy transfer involves two main assumptions about the intrinsic state functions, namely, $h_{l}(\mathbf{x}, t)$ and $h_{n l}(\mathbf{x}, t)$ as:

- The rate of change of the local contribution $\rho(\mathbf{x}) d V_{\mathbf{x}} \dot{h}_{l}(\mathbf{x}, t)$ depends on the thermal energy flux across the boundaries of the control volume $d V_{\mathbf{x}}$, namely, $\mathbf{q}(\mathbf{x}, t)$ as:

$$
\rho(\mathbf{x}) \dot{h}_{l}(\mathbf{x}, t)=-\nabla \cdot \mathbf{q}(\mathbf{x}, t)+\rho(\mathbf{x}) r(\mathbf{x}, t)
$$

with $r(\mathbf{x}, t)$ a specific thermal power source at location $\mathbf{x}$ as it may be easily withdrawn from the balance in Fig. 1(a).

- The rate of change of the non-local thermal energy contribution $\rho(\mathbf{x}) d V_{\mathbf{x}} \dot{h}_{n l}(\mathbf{x}, t)$ is obtained as the resultant of the twopoints exchange in Eq. (1), namely, $\mathcal{P}^{(n l)}(\mathbf{x}, \mathbf{y} ; t)$ (see Fig. 1(b)) yielding an additional thermal power source for the control volume $d V_{\mathbf{x}}$ :

$$
\rho(\mathbf{x}) d V_{\mathbf{x}} \dot{h}_{n l}(\mathbf{x}, t)=\int_{V_{\mathbf{y}}} \mathcal{P}^{(n l)}(\mathbf{x}, \mathbf{y} ; t) d V_{\mathbf{y}}=\rho(\mathbf{x}) d V_{\mathbf{x}} \int_{V_{\mathbf{y}}} \chi^{(n l)}(\mathbf{x}, \mathbf{y} ; t) \rho(\mathbf{y}) d V_{\mathbf{y}}
$$

The first principle of thermodynamics introduced by Eqs. (1) and (6) may be obtained substituting Eqs. (5) and (6) into the balance equation reported in Eq. (4) yielding:

$$
\rho(\mathbf{x}) \frac{\partial u(\mathbf{x}, t)}{\partial t}=-\nabla \cdot \mathbf{q}(\mathbf{x}, t)+\rho(\mathbf{x}) \int_{V_{\mathbf{y}}} \chi^{(n l)}(\mathbf{x}, \mathbf{y} ; t) \rho(\mathbf{y}) d V_{\mathbf{y}}+\rho(\mathbf{x}) r(\mathbf{x}, t)
$$

where the integral at the right-hand side is the non-local heat transfer contribute due to the interaction between the particle located at position $\mathbf{x}$ and all the other particles of the body [45].

The long-range contribution, namely function $\chi^{(n) l}(\mathbf{x}, \mathbf{y} ; t)$, is the long-range thermal energy transfer and it depends on the relative temperature measured at locations $\mathbf{x}$ and $\mathbf{y}$ as:

$$
\chi^{(n l)}(\mathbf{x}, \mathbf{y} ; t)=\kappa_{\alpha} g(\|\mathbf{x}-\mathbf{y}\|)[T(\mathbf{y}, t)-T(\mathbf{x}, t)]
$$

with $\kappa_{\alpha}$ a material dependent proportional coefficient and $g(\|\mathbf{x}-\mathbf{y}\|)$ is a distance-decaying function accounting for the decay of the long-range thermal energy transfer with the interdistance. In the following we assume that the function $g(\|\mathbf{x}-\mathbf{y}\|)$ decays as a power-law of the distance as:

$$
g(\|\mathbf{x}-\mathbf{y}\|)=\frac{1}{d_{n, l}(\bar{\alpha})} \frac{1}{\|\mathbf{x}-\mathbf{y}\|^{n+\alpha}}
$$

where $\alpha \in \mathbb{R}, n \in \mathbb{N}$ is the dimension of the topological space of the body (in our case $n=3$ ) and the normalization coefficient $d_{n, l}(\bar{\alpha})$ is related to the decaying exponent $\alpha$ and to the dimension of the topological space embedding the conductor $n$ 
(a)

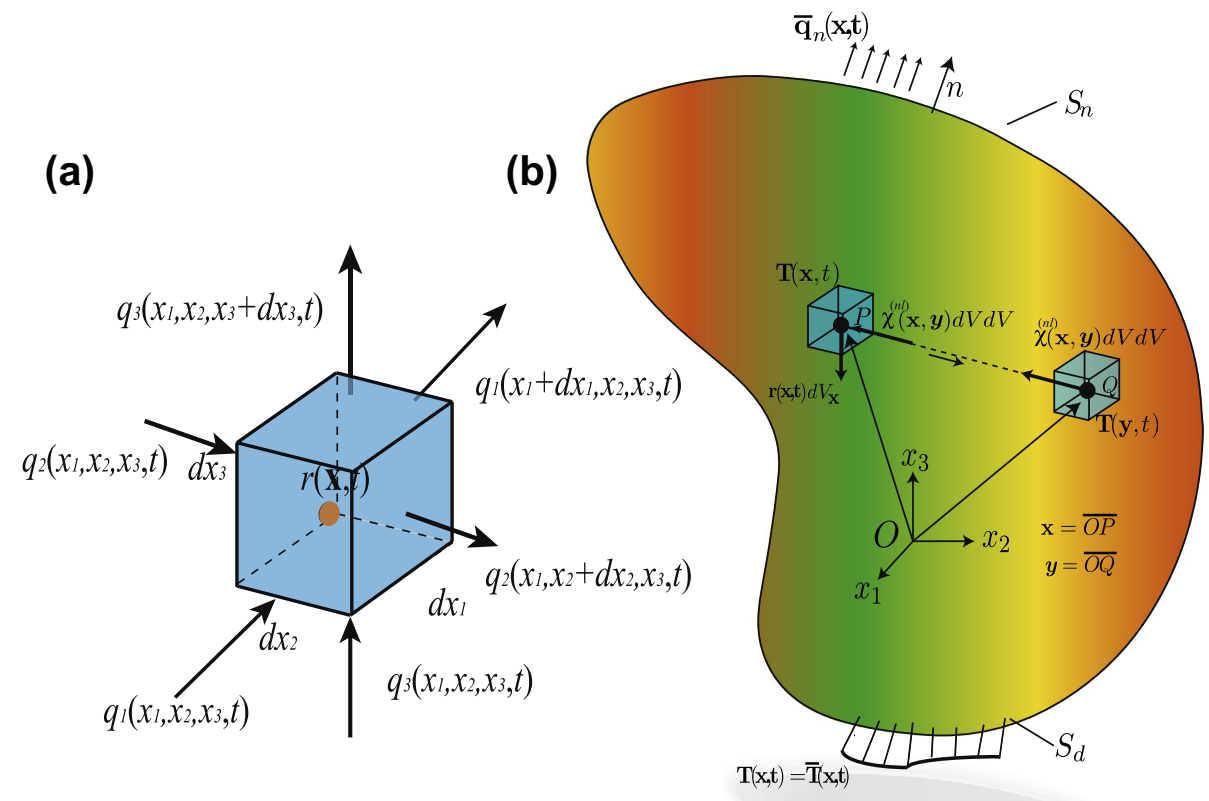

Fig. 1. (a) Balance among ingoing and outgoing thermal energy fluxes; (b) Ingoing and outgoing long-range thermal energy transfer for homogeneous conductor $(\rho(\mathbf{x})=\rho(\mathbf{y})=1)$.

(see Appendix A). Substituting Eq. (9) into Eq. (8), and assuming an homogeneous conductor $(\varrho(\mathbf{x})=\varrho(\mathbf{y})=\varrho)$ the internal energy balance reported in Eq. (7) is written in the form:

$$
\rho C_{V} \frac{\partial T}{\partial t}=-\nabla \cdot \mathbf{q}+\rho^{2} \kappa_{\alpha}\left(\hat{\mathrm{D}}_{\mathbf{x}}^{\alpha} T\right)+\rho r
$$

where $\hat{D}_{\mathbf{x}}^{\alpha} T$ is the integral contribution to the Marchaud fractional derivative of order $\alpha$, (see Appendix $A$ ), defined as:

$$
\hat{\mathrm{D}}_{\mathbf{x}}^{\alpha} T:=\frac{1}{d_{n, l}(\bar{\alpha})} \int_{V_{\mathbf{y}}} \frac{T(\mathbf{y}, t)-T(\mathbf{x}, t)}{\|\mathbf{x}-\mathbf{y}\|^{n+\alpha}} d V_{\mathbf{y}}
$$

when Fourier law for the classical local transfer of thermal energy is used, we obtain:

$$
\rho C_{V} \frac{\partial T}{\partial t}=\lambda \nabla^{2} T+\rho^{2} \kappa_{\alpha}\left(\hat{\mathrm{D}}_{\mathbf{x}}^{\alpha} T\right)+\rho r
$$

where $\lambda$ is the (local) heat conductivity and $\kappa_{\alpha}$ is the long-range transport coefficient, that we define anomalous heat conductivity. The temperature field may be obtained, in integral form, for any distributed heat sources $r(x, t)$ as we obtain the Greens' function for a concentrated temperature distribution $T_{0}(x)=T_{0} \delta(x)$.

The temperature field $T(\mathbf{x}, t)$ caused by a temperature pulse in unbounded domain is the solution of Eq. (12) replacing the integral term with its counterpart defined in unbounded domain, namely, $\left(\hat{\mathrm{D}}_{\mathbf{x}}^{\alpha} T\right) \rightarrow\left(\mathrm{D}_{\mathbf{x}}^{\alpha} T\right)$ that is the Marchaud fractional derivative. The initial and the integrability conditions associated to Eq. (12) for unbounded domains read:

$$
\begin{aligned}
& T(x, 0)=T_{0} \delta(x) ; \\
& T(-\infty, t)=T(\infty, t)=0
\end{aligned}
$$

yielding the Greens' function for the temperature distribution in Fourier domain, namely $\widetilde{T}(k, t)$, as the solution of the ordinary differential equation:

$$
\frac{d \widetilde{T}}{d t}=-\frac{\left(\lambda k^{2}+\rho \kappa_{\alpha}\|k\|^{\alpha}\right)}{\rho C_{V}} \widetilde{T}
$$

whose solution may be obtained by means of the inverse Fourier transform as: 


$$
T(x, t)=\frac{T_{0}}{2 \pi} \int_{-\infty}^{+\infty} e^{i k x} \widetilde{T}(k, t) d k=\frac{T_{0}}{2 \pi} \int_{-\infty}^{+\infty} e^{i k x} e^{\left(-\frac{\left(k k^{2}+\rho \kappa_{\alpha}\|k\|^{\alpha}\right) t}{\rho C_{V}}\right)} d k
$$

The temperature field corresponding to of the Fourier integral in Eq. (15) has been obtained in closed-form under the assumption of vanishing local contributions $(\lambda=0)$, for different decreasing values of the parameter $\alpha=1, \frac{3}{4}, \frac{1}{2}, \frac{1}{3}$, as reported in (Fig. 2). It may be observed that, if $\alpha=1$ then the Fourier integral in Eq. (15) reduces to a Cauchy-type temperature field as: $T(x, t)=\frac{2 t}{\left(t^{2}+x^{2}\right)}$ (Fig. 2(a)), whereas, for $\alpha=1 / 2$ a linear combination of Fresnel integrals, namely, $S(x)$ and $C(x)$ represents the temperature distribution reported in (Fig. 2(c)) as:

$$
T(x, t)=\frac{\sqrt{\frac{\pi}{2}} t\left(\operatorname{Cos}\left[\frac{t^{2}}{4 x}\right]\left(1-2 C(x)\left[\frac{t}{\sqrt{2 \pi} \sqrt{\|x\|}}\right]\right)+\left(1-2 S(x)\left[\frac{t}{\sqrt{2 \pi} \sqrt{\|x\|}}\right]\right) \operatorname{Sin}\left[\frac{t^{2}}{4\|x\|}\right]\right)}{\|x\|^{3 / 2}}
$$

In passing it must be stressed that a closed-form analytical solution for the fractional-order differential equation in Eq. (15) is obtained, as $\lambda=0, \forall \alpha \in \mathbb{Q}$ where $\mathbb{Q} \subset \mathbb{R}$ is the set of rational numbers.

\subsection{The Clausius-Planck relation with fractional-order heat transport}

The proposed model of thermal energy transfer in rigid conductors requires the introduction of appropriate transport relations among the state variables of the system: $(i)$ The flux $\mathbf{q}(\mathbf{x}, t)$ and the temperature field $T(\mathbf{x}, t)$; (ii) The long-range transfer $\chi^{(n l)}(\mathbf{x}, \mathbf{y}, t)$ and the relative temperature field $\Delta T(|\mathbf{x}-\mathbf{y}|, t)=T(\mathbf{y}, t)-T(\mathbf{x}, t)$.

The functional expression as well as the signs of the transport coefficients must be compliant with the second principle of thermodynamics expressed in terms of the well-known Clausius-Planck inequality. Indeed the use of the Clausius-Planck (or Clausius-Kelvin) inequality is equivalent to the use of the Clausius-Duhem form of the second principle of thermodynamics as the thermodynamics restrictions among the state variables have been fulfilled.
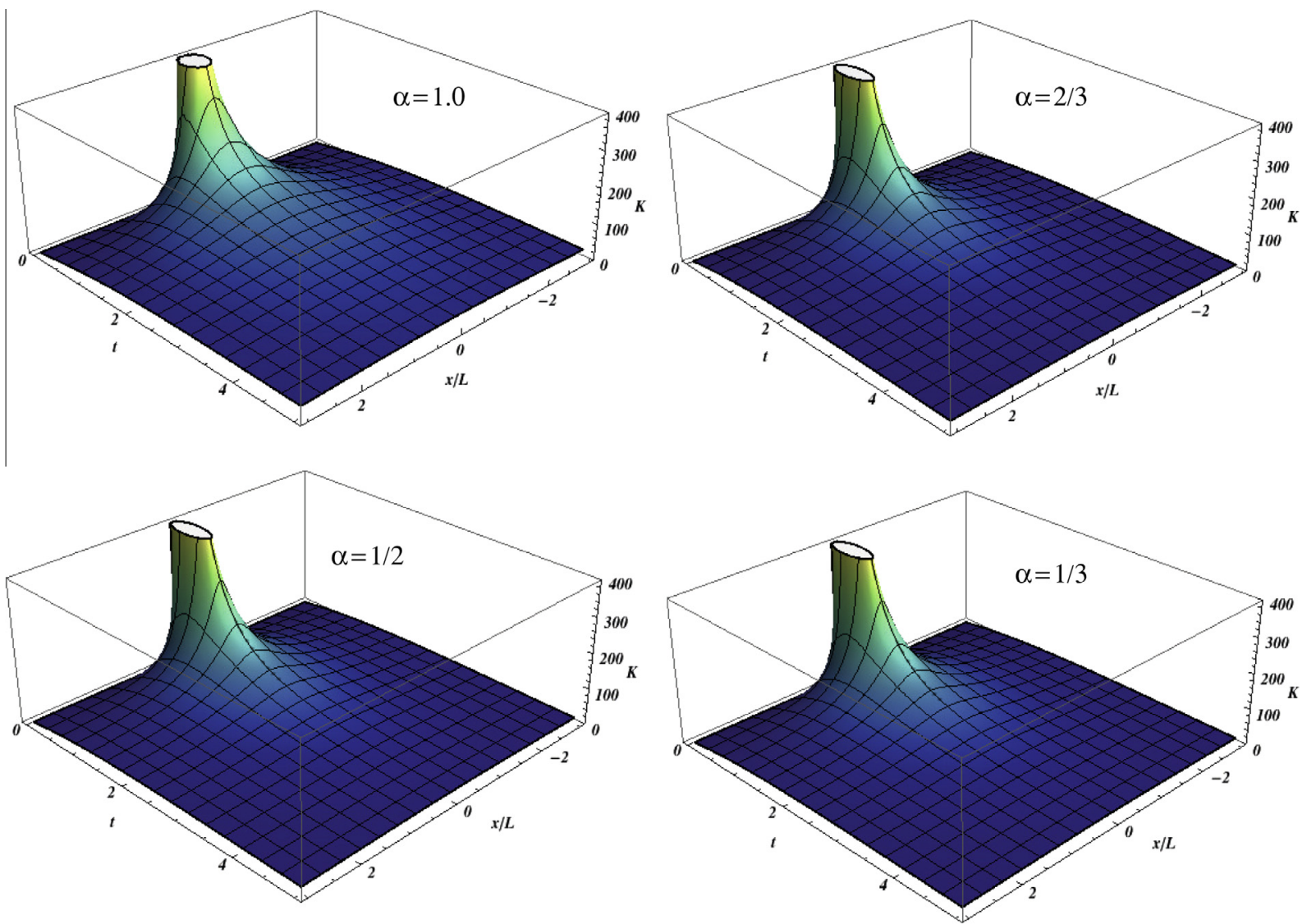

Fig. 2. Temperature distribution for diffusion of temperature pulse for $\left(\lambda=0, \kappa_{\alpha}=1.0, T_{0}=400 \mathrm{~K}\right)$ for different values of the differentiation order. 
The compatibility of the proposed model is assessed in terms of the entropy state function, namely, $s(\mathbf{x}, t)$ that must obey to Clausius-Planck inequality for a thermodynamical system evolving from state $A \rightarrow B$ at time instants $t_{A}$ and $t_{B}$, respectively, as:

$$
\triangle s_{A B}(\mathbf{x})=s\left(\mathbf{x}, t_{B}\right)-s\left(\mathbf{x}, t_{A}\right)=\int_{t_{A}}^{t_{B}} \rho \dot{s}(\mathbf{x}, t) d t \geqslant \int_{t_{A}}^{t_{B}} \frac{d h(\mathbf{x}, t)}{T(\mathbf{x}, t)}
$$

where we introduced the specific entropy function rate $\dot{s}(\mathbf{x}, t)$ and $d h$ is the thermal energy increment of the body subdomain at location $\mathbf{x}$. Recalling that, in a rigid body, $s=s(u)$ and that $\partial s / \partial u=1 / T$, making use of the energy balance equation, the right hand side of Eq. (17) may be written in the form:

$$
\rho \dot{s}(\mathbf{x}, t) \geqslant-\frac{\nabla \cdot \mathbf{q}(\mathbf{x}, t)}{T(\mathbf{x}, t)}+\frac{\rho(\mathbf{x}) r(\mathbf{x}, t)}{T(\mathbf{x}, t)}+\frac{\rho(\mathbf{x})}{T(\mathbf{x}, t)} \int_{V_{\mathbf{y}}} \rho(\mathbf{y}) \chi^{(n l)}(\mathbf{x}, \mathbf{y}, t) d V_{\mathbf{y}}
$$

The observation of Eq. (18) for the specific entropy rate increment shows that an additional contribution at the right-hand side is obtained with respect to the classical expression of the Clausius-Planck inequality. Eq. (18) may be recast in a more convenient form, introducing the specific entropy rate production $\sigma^{(s)}(\mathbf{x}, t) \geqslant 0$ for any thermodynamical process yielding:

$$
\rho \dot{s}(\mathbf{x}, t)+\frac{\nabla \cdot \mathbf{q}(\mathbf{x}, t)}{T(\mathbf{x}, t)}-\frac{\rho(\mathbf{x}) r(\mathbf{x}, t)}{T(\mathbf{x}, t)}-\frac{\rho(\mathbf{x})}{T(\mathbf{x}, t)} \int_{V_{\mathbf{y}}} \rho(\mathbf{y}) \chi^{(n l)}(\mathbf{x}, \mathbf{y}, t) d V_{\mathbf{y}}=\rho \sigma^{(s)}(\mathbf{x}, t) \geqslant 0
$$

On the other hand the entropy rate may be expressed at location $\mathbf{x}$ in the form of a balance among the incoming and the outcoming entropy flux in the unitary time so that:

$$
\rho \dot{s}(\mathbf{x}, t)=-\nabla \cdot \mathbf{J}_{l}^{(s)}(\mathbf{x}, t)+\int_{V_{\mathbf{y}}} J_{n l}^{(s)}(\mathbf{x}, \mathbf{y}, t) d V_{\mathbf{y}}+\frac{\rho(\mathbf{x}) r(\mathbf{x}, t)}{T(\mathbf{x}, t)}+\rho(\mathbf{x}) \sigma^{(s)}(\mathbf{x}, t)
$$

where we introduced the local and non-local long-range entropy transfer, respectively, $\mathbf{J}_{l}^{(s)}(\mathbf{x}, t)$ and $J_{n l}^{(s)}(\mathbf{x}, \mathbf{y}, t)$ related to the local and long-range thermal energy transfer, respectively.

Eq. (20) may be substituted into Eq. (18) to yield the inequality:

$$
-\frac{\nabla \cdot \mathbf{q}(\mathbf{x}, t)}{T(\mathbf{x}, t)}+\frac{\rho(\mathbf{x})}{T(\mathbf{x}, t)} \int_{V_{\mathbf{y}}} \rho(\mathbf{y}) \chi^{(n l)}(\mathbf{x}, \mathbf{y}, t) d V_{\mathbf{y}}+\nabla \cdot \mathbf{J}_{l}^{(s)}(\mathbf{x}, t)-\int_{V_{\mathbf{y}}} J_{n l}^{(s)}(\mathbf{x}, \mathbf{y}, t) d V_{\mathbf{y}} \geqslant 0
$$

The entropy flux is assumed to be a function of state of the local contribution to the internal energy rate $\mathbf{J}_{l}^{(s)}(\mathbf{x})=\varphi_{l}\left(u_{l}\right) \mathbf{q}_{\mathbf{l}}(\mathbf{x})$ and, by similar considerations we will assume that the long-range entropy transfer is provided as $J_{n l}^{(s)}(\mathbf{x}, \mathbf{y})=\varphi_{n l}\left(u_{n l}\right) Q^{2} \chi^{(n l)}(\mathbf{x}, \mathbf{y})$ (assuming $\rho(\mathbf{x})=\rho$ ). Under these circumstances the expression in Eq. (21), omitting the dependence on the time variabile $t$, yields:

$$
\left(\varphi_{l}\left(u_{l}\right)-\frac{1}{T(\mathbf{x})}\right) \nabla \cdot \mathbf{q}(\mathbf{x})+\mathbf{q}(\mathbf{x}) \cdot \nabla\left(\varphi_{l}\left(u_{l}\right)\right)-\int_{V_{\mathbf{y}}}\left(\varphi_{n l}\left(u_{n l}\right)-\frac{\varrho^{2} \chi^{(n l)}(\mathbf{x}, \mathbf{y})}{T(\mathbf{x})}\right) d V_{\mathbf{y}} \geqslant 0
$$

The inequality restriction in Eq. (22) leads to the conclusion that the linear term involving the thermal energy flux $\mathbf{q}(\mathbf{x}, \mathbf{t})$ must vanish yielding the relation:

$$
\varphi_{l}\left(u_{l}\right)=\frac{1}{T(\mathbf{x}, t)}
$$

that, upon substitution into Eq. (22) it yields:

$$
\frac{\mathbf{q}(\mathbf{x}, t)}{T^{2}(\mathbf{x}, t)} \cdot \nabla(T(\mathbf{x}, t))+\int_{V_{\mathbf{y}}}\left(\varphi_{n l}\left(u_{n l}\right)-\frac{1}{T(\mathbf{x}, t)}\right) \varrho^{2} \chi^{(n l)}(\mathbf{x}, \mathbf{y}, t) d V_{\mathbf{y}} \leqslant 0
$$

The inequality in Eq. (24) for the entropy production may be fulfilled if, for the first term, we assume a linear force-flux relation as:

$$
\mathbf{q}(\mathbf{x}, t)=-\lambda \nabla(T(\mathbf{x}, t))
$$

with $\lambda \geqslant 0$ that corresponds to Fourier relation, whereas, the second term at right hand side must involve the inverse of a temperature field $1 / T$ for dimensionality sake. As we assume that the long-range entropy flux function $\varphi_{n l}\left(u_{n l}\right)=1 / T(\mathbf{y}, t)$ since $J_{n l}^{(s)}(\mathbf{x}, \mathbf{y}, t)$ represents the entropy variation at location $\mathbf{x}$ due to a thermal source at location $\mathbf{y}$, then the integral term must satisfy the inequality:

$$
\int_{V_{\mathbf{y}}}\left(\frac{T(\mathbf{x}, t)-T(\mathbf{y}, t)}{T(\mathbf{x}, t) T(\mathbf{y}, t)}\right) \chi^{(n l)}(\mathbf{x}, \mathbf{y}, t) d V_{\mathbf{y}} \leqslant 0
$$

that is fulfilled if a linear force-flux relation for the long-range thermal energy transfer is assumed in Eq. (8): $\chi^{(n l)}(\mathbf{x}, \mathbf{y}, t)=g(\|\mathbf{x}-\mathbf{y}\|)[T(\mathbf{y}, t)-T(\mathbf{x}, t)]$ and $g(\|\mathbf{x}-\mathbf{y}\|, t) \geqslant 0$ as in Eq. (9). Such restrictions for the local and non-local ther- 
mal energy exchanges are satisfied with the power-law decaying function $g(\mathbf{x}, \mathbf{y})$ defined in Eq. (9) that is compliant with the second principle of thermodynamics [47].

\subsection{Numerical simulation: temperature distribution in $1 D$ rigid conductor}

The field of temperature distribution $T(\mathbf{x}, t)$ ruled by Eq. (13) in a bounded 1D domain of length $L$ is provided by the solution of the fractional differential equation that reads (omitting arguments):

$$
\rho C_{V} \frac{\partial T}{\partial t}=\lambda \nabla^{2} T+\rho \kappa_{\alpha}\left(\hat{\mathbf{D}}_{x}^{\alpha} T\right)
$$

where only the integral parts of the Marchaud fractional derivatives are involved. In this case the analysis of the temperature field is obtained resorting to the fractional finite difference (FFD) discretization of fractional derivative operator. Indeed, as we introduce a discrete grid of abscissas $x_{j}=(j-1) \Delta x$, with step $\Delta x=L /(N+1)$ the finite difference solution of the non-local temperature field will be obtained at the gridpoints $x_{j},(j=1,2, \ldots, N+1)$ introducing the central finite difference operators for the second-order gradient and the FFD approximation of the D-Riesz fractional derivative as:

$$
\begin{aligned}
\frac{\partial^{2} f\left(x_{j}\right)}{\partial x^{2}} \backsim \frac{\Delta^{2} f_{j}}{\Delta x^{2}}=\frac{f\left(x_{j+1}\right)-2 f\left(x_{j}\right)+f\left(x_{j-1}\right)}{\Delta x^{2}} & \\
\left(\hat{\mathrm{D}}_{x}^{\alpha} f\right)\left(x_{j}\right) \simeq \Delta_{x}^{\alpha} f\left(x_{j}\right)= & \frac{\alpha^{-1}}{\Gamma(1-\alpha)}\left\{\sum_{h=1}^{j-1}\left[\left(x_{j-h+1}\right)^{-\alpha}-\left(x_{j-h}\right)^{-\alpha}\right] f\left(x_{h}\right)\right\} \\
& +\frac{\alpha^{-1}}{\Gamma(1-\alpha)}\left\{\sum_{h=j+1}^{N+1}\left[\left(x_{h-j}\right)^{-\alpha}-\left(x_{h-j-1}\right)^{-\alpha}\right] f\left(x_{h}\right)\right\}
\end{aligned}
$$

Introduction of the finite difference scheme in the governing equation of the temperature field in Eq. (28) yields a set of ordinary differential equations in time domain in the form:

$$
\dot{\mathbf{T}}(t)+\left[\mathbf{K}^{(l)}+\mathbf{K}^{(n l)}\right] \mathbf{T}(t)=\bar{r}(t)
$$

where $\mathbf{T}(t)=\left[\begin{array}{lllll}T_{1}(t) & T_{2}(t) & \ldots & \ldots & T_{N+1}(t)\end{array}\right]^{T}$ is a vector collecting the values of the temperature field at the grid points with initial conditions in the form $\mathbf{T}(0)=\mathbf{T}_{\mathbf{0}}$ and $\overline{\mathbf{r}}(t)$ is a vector gathering the values of the heat source in the conductor domain with elements $\bar{r}_{j}(t)=r\left(x_{j}, t\right) / C_{V}$. The $(N+1) \times(N+1)$ local and non-local diffusion matrices reported in Eq. (30) read, respectively:

$$
\begin{gathered}
\mathbf{K}^{(l)}=\frac{\lambda}{C_{V}(\Delta x)^{2}}\left[\begin{array}{cccccc}
1 & -1 & 0 & \ldots & \ldots & 0 \\
-1 & 2 & -1 & \ldots & \ldots & 0 \\
\ldots & \ldots & \ldots & \ldots & \ldots & \ldots \\
\ldots & \ldots & \ldots & \ldots & \ldots & \ldots \\
0 & \ldots & 0 & -1 & 2 & -1 \\
0 & 0 & \ldots & \ldots & -1 & 1
\end{array}\right] \\
\mathbf{K}^{(n l)}=\frac{\kappa_{\alpha} \alpha}{C_{V} \Gamma(1-\alpha)}\left[\begin{array}{cccccc}
K_{11}^{(n l)} & K_{12}^{(n l)} & K_{13}^{(n l)} & \ldots & \ldots & K_{1 N+1}^{(n l)} \\
\operatorname{Sym} & K_{22}^{(n l)} & K_{23}^{(n l)} & \ldots & \ldots & K_{2 N+1}^{(n l)} \\
\ldots & \ldots & \ldots & \ldots & \ldots & \ldots \\
\ldots & \ldots & \ldots & \ldots & \ldots & \ldots \\
\operatorname{Sym} & & & \ldots & K_{N N}^{(n l)} & \ldots \\
\operatorname{Sym} & \ldots & \ldots & \ldots & S y m & K_{N+1 N+1}^{(n l)}
\end{array}\right]
\end{gathered}
$$

where $K_{j h}^{(n l)}=\left(x_{j-h+1}\right)^{-\alpha}-\left(x_{j-h}\right)^{-\alpha}$ and with the diagonal terms in Eq. (32) that read $K_{j j}^{(n l)}=-\sum_{h \neq j}^{N+1} K_{h=1}^{(n l)}$. The differential equation reported in Eq. (30) has been solved with the approximate integration scheme provided by FFD for constant temperatures at the boundaries as:

$$
T(-L / 2, t)=T_{2}=200 \mathrm{~K} \quad T(L / 2, t)=T_{1}=100 \mathrm{~K}
$$

with initial conditions provided as a linear distributions of temperatures among the values $T_{2}$ and $T_{1}$ as:

$$
T(x, 0)=T_{2}-\frac{(1+2 x / L)\left(T_{2}-T_{1}\right)}{2}
$$

The corresponding numerical solution of the boundary value problem reported in Eq. (27) and in Eqs. (33) and (34) has been reported in (Fig. 3) for different values of the differentiation index $\alpha$. It may be observed that, introducing long-range thermal 

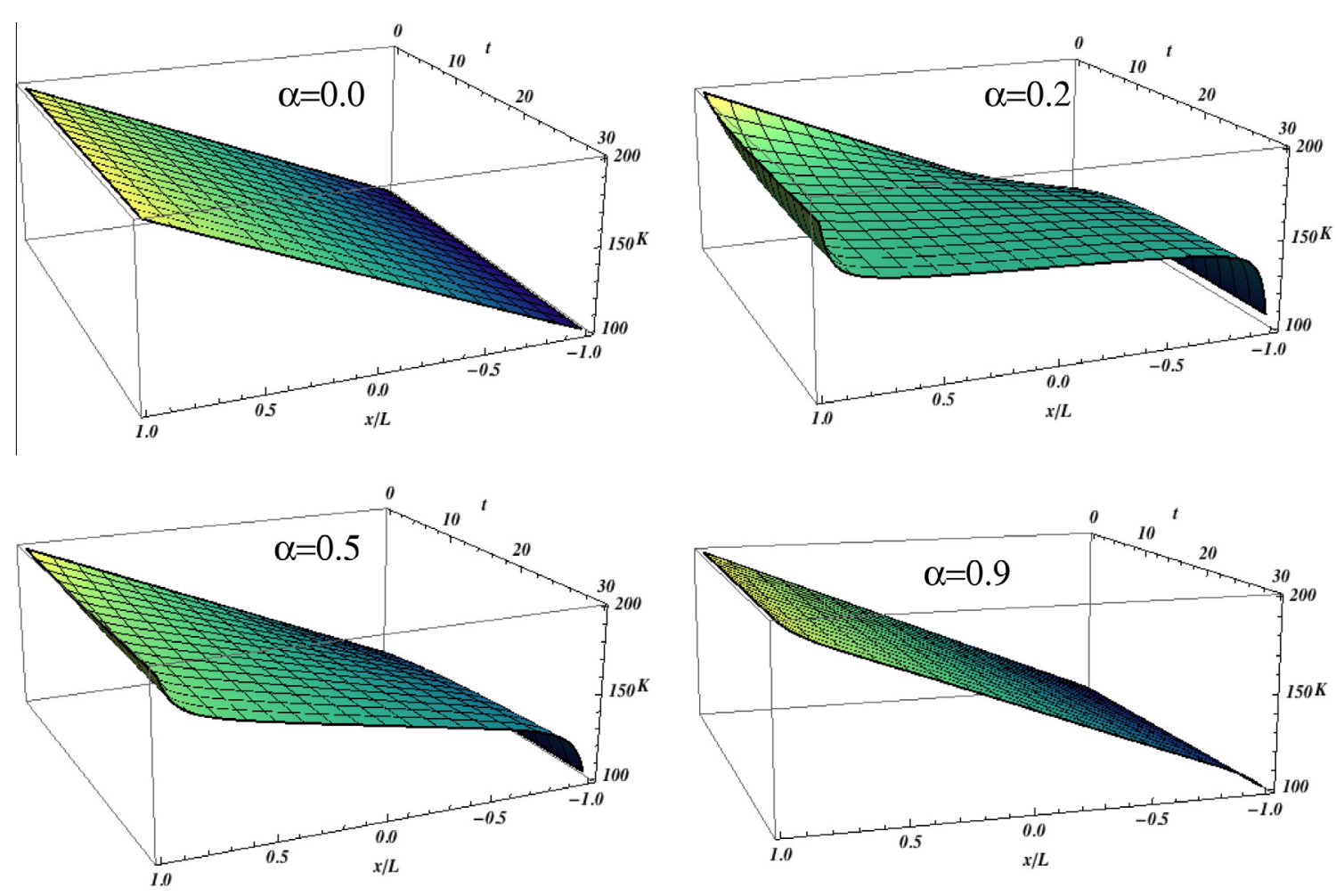

Fig. 3. Temperature distribution for different decay of long-range thermal energy transfer $\left(\lambda=10.0, \kappa_{\alpha}=0.1, C_{V}=1.0\right)$.

energy transfer, the time-evolution of the initial linear temperature field in the conductor shows high temperature gradients at the insulated borders of the 1D conductor as observed in Kapitsa effect. This feature is very interesting in thermal energy transport in nanowires and nanosheets since insulated and/or thermostated borders of the conductor involves propagation of phononic carriers. The propagation of energy at the subatomic scales involves modification in the state of electron density of the material and, henceforth, it induces a modification of the thermal conductivity of the material close to the borders [1,2].

The presence of phononic transport has been captured in this section with a diffusive equation and, henceforth, the instantaneous perturbation of the temperature field is transferred across the conductor with unbounded speed. Therefore there are no transitory regimes among the initial and the steady-state temperature field since no temperature waves are involved in the analysis. Such a consideration is further investigated in the next section in the context of non-equilibrium thermodynamics.

\section{A non-equilibrium model of fractional-order thermal energy transport}

The non-local model of thermal energy transfer used in previous section relies on the assumption of local thermodynamic equilibrium conditions. This assumption is appropriate for usual engineering applications but, as thermal energy transport involves high frequency processes with oscillation periods comparable with the relaxation times of phonons carrying thermal energy, an appropriate extension of Fourier transport equations must be accounted for.

The usual representation of the memory effect due to thermal energy flux is provided by additional contributions in the transport equations in terms of the first-order time derivative of the heat flux $\dot{\mathbf{q}}(\mathbf{x}, t)$ as in Maxwell-Cattaneo [42] model or resorting to more recent real-order derivatives $\left(D_{0^{+}}^{\alpha} \mathbf{q}\right)_{t}(\mathbf{x}, t)$ (see e.g. [5]). In this section the first-order relaxation of thermal energy transfer by means of the Cattaneo model for the local thermal energy exchange is considered as:

$$
\tau \dot{\mathbf{q}}(\mathbf{x}, t)+\mathbf{q}(\mathbf{x}, t)=-\lambda \nabla \mathrm{T}(\mathbf{x}, t)
$$

with $\tau \geqslant 0$ a material dependent relaxation time ruling the decay of the propagating thermal waves in the conductor.

Introducing the gradient operator $\nabla[\bullet]$ to both sides of Eq. (35) the following relation is obtained:

$$
\tau \frac{\partial}{\partial t}[\nabla \cdot \mathbf{q}(\mathbf{x}, t)]+[\nabla \cdot \mathbf{q}(\mathbf{x}, t)]=-\lambda \nabla^{2} \mathrm{~T}(\mathbf{x}, t)
$$

and, as we replace the divergence of the thermal energy flux in Eq. (35) with the corresponding expression in terms of temperature gradients as reported in Eq. (8) we get the 3D counterpart of the temperature equation yet obtained for the 1D case [45], in the form: 


$$
\tau \rho C_{V} \frac{\partial^{2} T(\mathbf{x}, t)}{\partial t^{2}}+\frac{\partial}{\partial \mathrm{t}}\left(\rho C_{V} T(\mathbf{x}, t)-\tau \mathrm{k}_{\alpha}\left(\hat{\mathbf{D}}^{\alpha} \mathrm{T}(\mathbf{x}, t)\right)\right)=\lambda \nabla^{2} \mathrm{~T}(\mathbf{x}, t)+\mathrm{k}_{\alpha}\left(\hat{\mathbf{D}}^{\alpha} T(\mathbf{x}, t)\right)+\rho r(\mathbf{x}, t)+\dot{\rho} r(\mathbf{x}, t)
$$

The use of Eq. (35) for local thermal energy transfer $\mathbf{q}(\mathbf{x}, t)$ involves non-monotonic increments of the entropy function $s=s(u)$ of CIT introduced in previous section as observed in previous papers [39,40]. Previous consideration leads to assess the thermodynamical consistency of the model in terms of the extended irreversible thermodynamics (EIT) as it is shown in the following section.

\subsection{The Clausius-Planck relation of EIT with fractional-order heat transport}

The introduction of a first-order time lag in the force-flux relation (Eq. (35)), requires the introduction of a non-equilibrium entropy, functionally dependent on the internal energy density $u$ and on the thermal energy flux $\mathbf{q}$ in the form: $s=s(u, \mathbf{q})$. Under this assumption the local entropy flux shows the functional dependence $\mathbf{J}_{l}^{(s)}=\mathbf{J}_{l}^{(s)}(u, \mathbf{q})=\varphi(u) \mathbf{q}[28]$. In this setting, the non-equilibrium entropy rate reads:

$$
\dot{s}=\dot{s}(u, \mathbf{q})=\left(\frac{\partial s}{\partial u}\right)_{\mathbf{q}} \dot{u}+\left(\frac{\partial s}{\partial \mathbf{q}}\right)_{u} \dot{\mathbf{q}}
$$

In the following we assume an isotropic body so that the functional dependence entropy function and local entropy flux may be assumed as: $s=s(u, \mathbf{q} \cdot \mathbf{q})=s\left(u, q^{2}\right)$ and: $\mathbf{J}_{l}^{(s)}=\mathbf{J}_{l}^{(s)}\left(u, q^{2}\right)$, respectively. Under these circumstances the entropy rate function in Eq. (38) may be written as:

$$
\dot{s}=\dot{s}\left(u, q^{2}\right)=\left(\frac{\partial s}{\partial u}\right)_{q^{2}} \dot{u}+\left(\frac{\partial s}{\partial q^{2}}\right)_{u} 2 \mathbf{q} \cdot \dot{\mathbf{q}}
$$

Substitution of Eq. (39) into the entropy balance equation reported in Eq. (27) yields (omitting the dependence on the time variable):

$$
\rho(\mathbf{x})\left(\frac{\partial s}{\partial u}\right)_{q^{2}} \dot{u}+\rho\left(\frac{\partial s}{\partial q^{2}}\right)_{u} 2 \mathbf{q} \cdot \dot{\mathbf{q}}+\nabla \cdot \mathbf{J}_{l}^{(s)}(\mathbf{x})-\int_{V_{\mathbf{y}}} J_{n l}^{(s)}(\mathbf{x}, \mathbf{y}) d V_{\mathbf{y}}-\frac{\rho r}{T(\mathbf{x})}=\rho(\mathbf{x}) \sigma^{(s)}(\mathbf{x}, t)
$$

As we substitute, for the rate of internal energy, the expression in terms of the local and long-range thermal energy fluxes in Eq. (8) and accounting for Cattaneo transport equation in Eq. (35), after straightforward manipulations we get:

$$
\begin{aligned}
& \left(\frac{\partial s}{\partial u}\right)_{q^{2}}\left(-\nabla \cdot \mathbf{q}+\rho(\mathbf{x}) \int_{V_{\mathbf{y}}} \rho(\mathbf{y}) \chi^{(n l)}(\mathbf{x}, \mathbf{y}) d V_{\mathbf{y}}+\rho r\right)+\rho\left(\frac{\partial s}{\partial q^{2}}\right)_{u} 2 \mathbf{q} \cdot \dot{\mathbf{q}}+\nabla \cdot \mathbf{J}_{l}^{(s)}(\mathbf{x})-\int_{V_{\mathbf{y}}} J_{n l}^{(s)}(\mathbf{x}, \mathbf{y}) d V_{\mathbf{y}}-\frac{\rho(\mathbf{x}) r}{T(\mathbf{x})} \\
& \quad=\rho(\mathbf{x}) \sigma^{(s)}(\mathbf{x}, t)
\end{aligned}
$$

The derivative of the entropy function with respect to the internal energy, $\left(\frac{\partial s}{\partial u}\right)_{q^{2}}$, is, dimensionally, a temperature field and under the assumption in Eq. (38) it may be selected as the absolute temperature of the body: $\left(\frac{\partial s}{\partial u}\right)_{q^{2}}=\frac{1}{T(\mathbf{x})}$ yielding Eq. $(41)$ in the form:

$$
-\frac{\nabla \cdot \mathbf{q}}{T(\mathbf{x})}+\frac{\rho(\mathbf{x})}{T(\mathbf{x})} \int_{V_{\mathbf{y}}} \rho(\mathbf{y}) \chi^{(n l)}(\mathbf{x}, \mathbf{y}) d V_{\mathbf{y}}-\rho(\mathbf{x})\left(\frac{\partial s}{\partial q^{2}}\right)_{u} 2 \mathbf{q} \cdot\left(\frac{\lambda}{\tau} \nabla T+\frac{\mathbf{q}}{\tau}\right)+\nabla \cdot \mathbf{J}_{l}^{(s)}(\mathbf{x})-\int_{V_{\mathbf{y}}} J_{n l}^{(s)}(\mathbf{x}, \mathbf{y}) d V_{\mathbf{y}}=\rho(\mathbf{x}) \sigma^{(s)}(\mathbf{x}, t)
$$

Right-hand side of Eq. (42), namely entropy production rate, must be positive, so that, after some manipulation, the following inequality reads:

$$
\begin{aligned}
\left(\varphi_{l}-\frac{1}{T(\mathbf{x})}\right) \nabla \cdot \mathbf{q}- & \int_{V_{\mathbf{y}}}\left(J_{n l}^{(s)}(\mathbf{x}, \mathbf{y})-\frac{\rho(\mathbf{x}) \rho(\mathbf{y}) \chi^{(n l)}(\mathbf{x}, \mathbf{y})}{T(\mathbf{x})}\right) d V_{\mathbf{y}}-\frac{2 \rho(\mathbf{x}) \lambda}{\tau}\left(\frac{\partial s}{\partial q^{2}}\right) 2 \mathbf{q} \cdot(\nabla T) \\
& -\frac{2 \boldsymbol{\rho}(\mathbf{x})}{\tau}\left(\frac{\partial s}{\partial q^{2}}\right) q^{2}+\left(\nabla \varphi_{l}\right) \cdot \mathbf{q} \geqslant 0
\end{aligned}
$$

where $\mathbf{J}_{l}^{(s)}=\varphi_{l}(u) \mathbf{q}$. The condition in Eq. (43) may be respected as function $\varphi_{l}(u)=\frac{1}{T(\mathbf{x})}$ yielding the following inequality for the remaining terms:

$$
\int_{V_{\mathbf{y}}}\left(J_{n l}^{(s)}(\mathbf{x}, \mathbf{y})-\frac{\rho^{2} \chi^{(n l)}(\mathbf{x}, \mathbf{y})}{T(\mathbf{x})}\right) d V_{\mathbf{y}}+\left(\frac{2 \rho \lambda}{\tau} \mathbf{q} \cdot(\nabla T)+\frac{2 \boldsymbol{\rho}}{\tau} q^{2}\right)\left(\frac{\partial s}{\partial q^{2}}\right)+\frac{\nabla T(\mathbf{x})}{T(\mathbf{x})^{2}} \cdot \mathbf{q} \leqslant 0
$$

that must be fulfilled for any thermodynamical process in the body.

The first term in Eq. (44) is analogous to Eq. (24) and assuming $J_{n l}^{(s)}(\mathbf{x}, \mathbf{y})=\varphi_{n l}(u) \rho(\mathbf{x}) \rho(\mathbf{y}) \chi^{(n l)}(\mathbf{x}, \mathbf{y})$ the condition on the proportionality function $\varphi_{n l}(u)$ yields: $\varphi_{n l}(u)=\frac{1}{T(\mathbf{y})}$ to respect the Clausius-Planck inequality in Eq. (44) for any thermodynamical process. As a consequence the decaying function $g(\mathbf{x}, \boldsymbol{\xi}) \geqslant 0$ as in previous section. The second term in Eq. (44) satisfies the inequality sign as: 


$$
\left(\frac{2 \rho \lambda}{\tau}\left(\frac{\partial s}{\partial q^{2}}\right)+\frac{1}{T(\mathbf{x})^{2}}\right) \mathbf{q} \cdot(\nabla T)+\frac{2 \rho}{\tau}\left(\frac{\partial s}{\partial q^{2}}\right) q^{2} \leqslant 0
$$

that may be fulfilled only as the first-order derivative of the extended entropy function $s=s\left(u, q^{2}\right)$ reads:

$$
\left(\frac{\partial s}{\partial q^{2}}\right)=-\frac{\tau}{2 \rho \lambda} \frac{1}{T(\mathbf{x})^{2}}
$$

yielding a thermodynamical restriction upon the sign of the thermal conductivity $\lambda \geqslant 0$ as in Fourier transport equation that has been considered in previous section.

\subsection{Numerical simulation: temperature distribution in $1 D$ domain}

The temperature equation for ballistic thermal energy transfer in presence of pure diffusion (Eq. (13)), or with mixed/ballistic diffusion thermal energy transfer (Eq. (36)) may be solved with Fourier transform method. Initially we confine the analysis to the temperature distribution in 1D unbounded domain, neglecting the presence of internal heat sources $r(x, t)=\dot{r}(x, t)=0$, that is ruled by the initial value problem:

$$
C_{V} \boldsymbol{\rho} \tau \frac{\partial^{2} T(\mathrm{x}, t)}{\partial t^{2}}+\frac{\partial}{\partial t}\left(C_{V} \rho T(\mathrm{x}, t)-\tau \kappa_{\alpha}\left(\mathbf{D}^{\alpha} \mathrm{T}(\mathrm{x}, t)\right)\right)=\lambda \nabla^{2} \mathrm{~T}(\mathrm{x}, t)+\kappa_{\alpha}\left(\mathbf{D}^{\alpha} \mathrm{T}(\mathrm{x}, t)\right) \quad T(x .0)=\frac{T_{0} e^{-\frac{x^{2}}{4 \sigma^{2}}}}{\sqrt{2 \pi} \sigma} ; \quad \dot{T}(x, 0)=0
$$

Fourier transform of Eq. (47) reads:

$$
\frac{d^{2} \widetilde{T}(\kappa, t)}{d t^{2}}+\frac{d \widetilde{T}(\kappa, t)}{d t}\left(\frac{1-\tau \Lambda_{\alpha}\left\|\kappa^{\alpha}\right\|}{\tau}\right)+\left(\frac{\lambda \kappa^{2}-\Lambda_{\alpha}\left\|\kappa^{\alpha}\right\|}{\tau}\right) \widetilde{T}(\kappa, t)=0 \quad \hat{T}(x .0)=T_{0} e^{-\kappa^{2} \sigma^{2}} ; \quad \dot{\hat{T}}(x, 0)=0
$$

with $C_{\alpha} \cos (\alpha \pi / 2) / \varrho=\Lambda_{\alpha}$. We define the coefficients in Eq. (48) as:

$$
A(\kappa)=\frac{1-\tau \Lambda_{\alpha}\|\kappa\|^{\alpha}}{2 \tau} ; \quad B(\kappa)=\frac{\lambda \kappa^{2}-\tau \Lambda_{\alpha}\|\kappa\|^{\alpha}}{\tau}
$$

yielding the reduced version of the differential equation in Eq. (48) as:
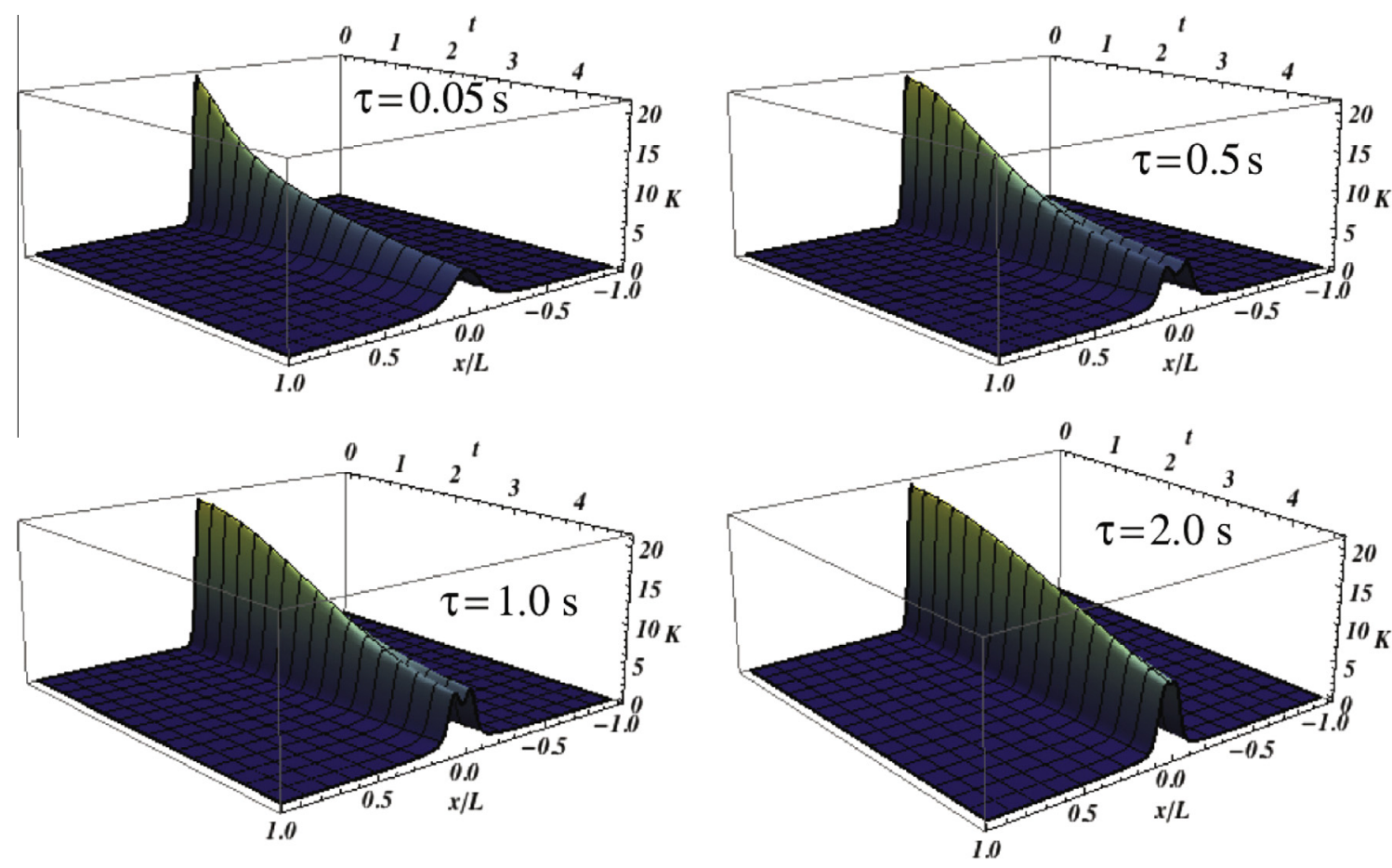

Fig. 4. Propagation of a Gaussian temperature pulse with $T_{0}=20 \mathrm{~K}, \sigma=0.1$ for different values of the time lag $\tau$ with $\left(\lambda=10.0, \kappa_{\alpha}=0.1, \alpha=0.3\right.$, $C_{V}=1.0$ ). 


$$
\frac{\mathrm{d}^{2} \widetilde{T}(\kappa, t)}{\mathrm{d} t^{2}}+A(\kappa) \frac{d \widetilde{T}(\kappa, t)}{\mathrm{d} t}+B(\kappa) \widetilde{T}(\kappa, t)=0 \quad \hat{T}(x, 0)=T_{0} e^{-\kappa^{2} \sigma^{2}} ; \quad \dot{\hat{T}}(x, 0)=0
$$

whose solution is provided as usual linear combination of exponential functions with coefficients dependent on the initial conditions. In this regard the temperature distribution is provided, in Fourier domain as:

$$
T(\mathrm{x}, t)=\frac{T_{0}}{2 \pi} \int_{-\infty}^{+\infty} \frac{r_{2}(\kappa) e^{r_{1}(\kappa) t}-r_{1}(\kappa) e^{r_{2}(\kappa) t}}{r_{1}(\kappa)-r_{2}(\kappa)} e^{-\kappa^{2} \sigma^{2}} e^{i \kappa x} d \kappa
$$

where $r_{1}(\kappa)$ and $r_{2}(\kappa)$ are the solution of the characteristic equation expressed in terms of the coefficients $A(\kappa)$ and $B(\kappa)$ as:

$$
r_{1}(\kappa)=-\left(A(\kappa)+\sqrt{A(\kappa)^{2}-B(\kappa)}\right) ; \quad r_{2}(\kappa)=-\left(A(\kappa)-\sqrt{A(\kappa)^{2}-B(\kappa)}\right)
$$

The presence of time lag $\tau$ in thermal energy transport induces the propagation of a decaying thermal wave that is strongly influenced by the fractional-order transport represented by integral terms in Eq. (50). The effects of the fractional differentiation order in the temperature distribution is reported in (Fig. 4(a)-(d)) showing the presence of a thermal wave propagating in 1D domain with a faster decay induced by the long-range diffusive transport. The effects induced by the time lag $\tau$ in the temperature distribution of local and non-local type is showed in (Figs. 5(a)-(d)) reporting the temperatures of a 1D domain for different values of the time lag $(\tau=0 ; \tau=0.05 \mathrm{~s} ; \tau=0.5 \mathrm{~s} ; \tau=5 \mathrm{~s}$ ). It is shown that, as time lag increases, some of the thermal energy is transferred by diffusion (smaller values of $\tau$ ) among adjacent and non-adjacent locations whereas some other is transferred by ballistic motion of thermal phonons. In case of larger values of the time lags, the presence of damped thermal waves corresponding to ballistic motion increases over classical diffusion (5(c)-(d)).

The study of the temperature field in an 1D bounded domain is ruled by the second-order integro-differential equation containing the integral parts of the Marchaud derivatives as:

$$
C_{V} \boldsymbol{\rho} \tau \frac{\partial^{2} T(\mathrm{x}, t)}{\partial t^{2}}+\frac{\partial}{\partial t}\left(C_{V} \rho T(\mathrm{x}, t)-\tau \kappa_{\alpha}\left(\hat{\mathbf{D}}^{\alpha} T(\mathrm{x}, t)\right)\right)=\lambda \nabla^{2} T(\mathrm{x}, t)+\kappa_{\alpha}\left(\hat{\mathbf{D}}^{\alpha} T(\mathrm{x}, t)\right)
$$

The numerical analyses reported in the paper aim to highlight the effects of the time-lag $\tau$ and of the coefficient $\alpha$ on the temperature field as well as on the propagation of thermal waves induced by initial disturbances. The temperature field has been obtained resorting to the FFD scheme introducing the discretization grid with $N+1$ node and step $\Delta x=\frac{L}{(N+1)}$ the temperature values at the nodes, namely, $T_{j}(t)$ with $j=1,2, \ldots N+1$ are provided as solution of the differential equations:

$$
\tau \ddot{\mathbf{T}}(t)+\left[\mathbf{C}^{(l)}+\mathbf{C}^{(n l)}\right] \dot{\mathbf{T}}(t)+\left[\mathbf{K}^{(l)}+\mathbf{K}^{(n l)}\right] \mathbf{T}(t)=\overline{\mathbf{r}}(t)
$$
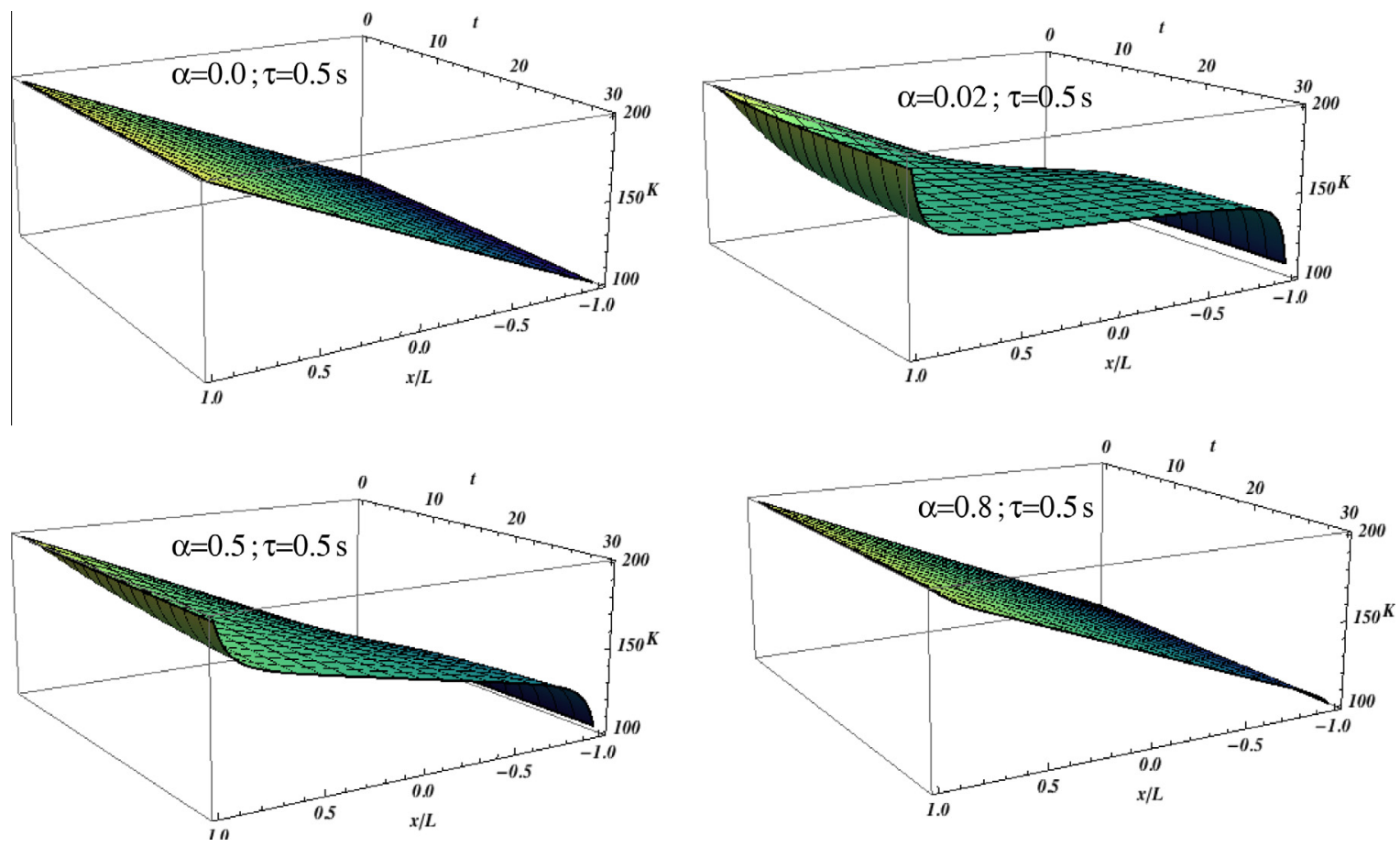

Fig. 5. Temperature distribution with long-range thermal energy transfer for different values of the differentiation order $\alpha\left(\lambda=10.0, \kappa_{\alpha}=0.1, C_{V}=1.0\right)$. 

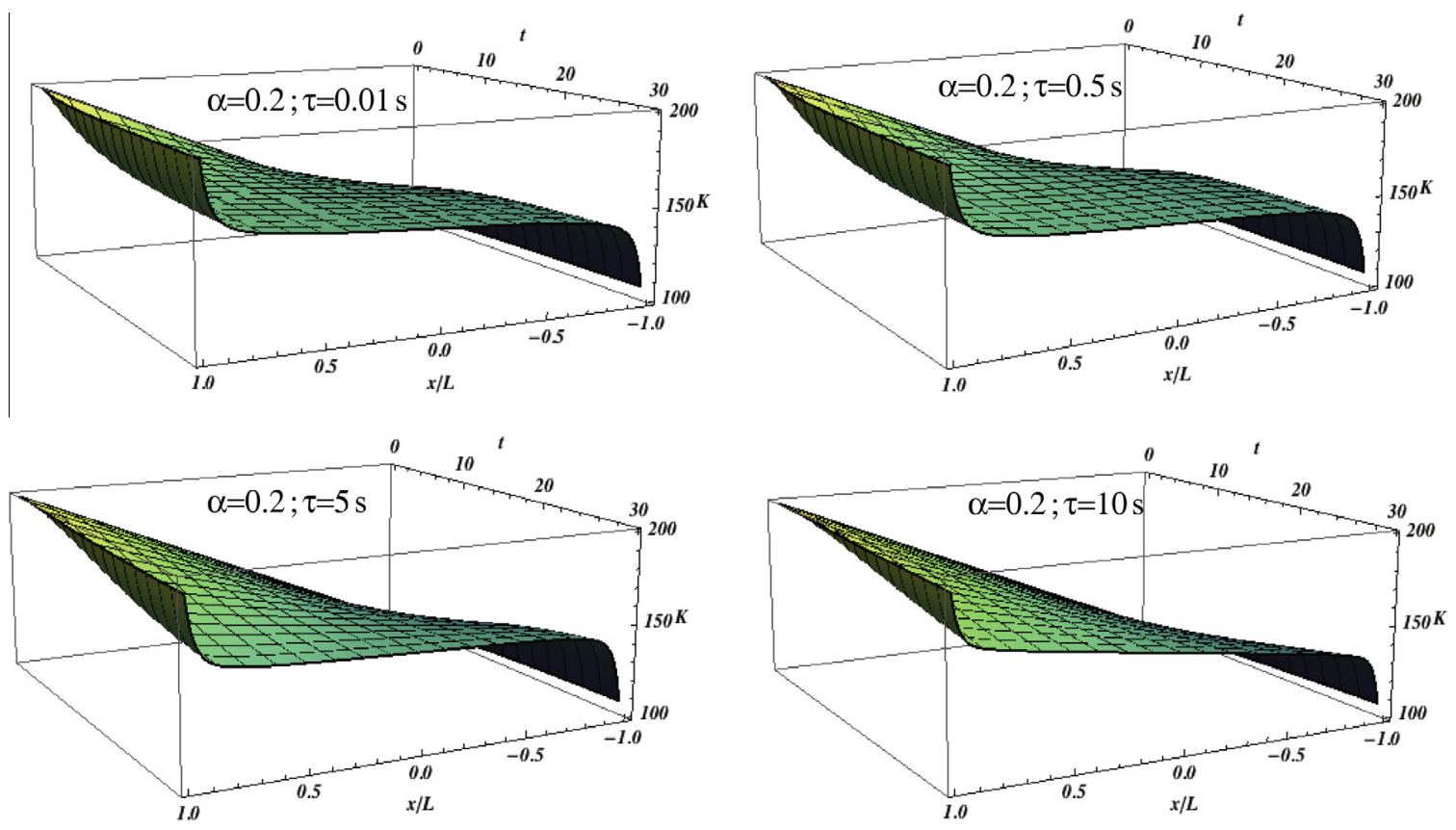

Fig. 6. Temperature distribution with long-range thermal energy transfer for different values of the time lag $\left(\lambda=10.0, \kappa_{\alpha}=0.1, C_{V}=1.0\right)$.

where the coefficient matrices $\mathbf{K}^{(l)}$ and $\mathbf{K}^{(n)}$ have been reported in Eqs. (33) and (34), respectively. The local coefficient matrix $\mathbf{C}^{(l)}$ coincides with the $(N+1)$ identity matrix $\mathbf{C}^{(l)}=\mathbf{I}$ and non-local dissipation matrices $\mathbf{C}^{(n l)}$, reads, respectively:

$$
\mathbf{C}^{(n l)}=\frac{\tau \kappa_{\alpha} \alpha}{C_{V} \Gamma(1-\alpha)}\left[\begin{array}{cccccc}
C_{11}^{(n l)} & C_{12}^{(n l)} & C_{13}^{(n l)} & \ldots & \ldots & C_{1 N+1}^{(n l)} \\
S y m & C_{22}^{(n l)} & C_{23}^{(n l)} & \ldots & \ldots & C_{2 N+1}^{(n)} \\
\ldots & \ldots & \ldots & \ldots & \ldots & \ldots \\
\ldots & \ldots & \ldots & \ldots & \ldots & \ldots \\
S y m & & & \ldots & C_{N N}^{(n)} & \ldots \\
S y m & \ldots & \ldots & \ldots & S y m & C_{N+1 N+1}^{(n)}
\end{array}\right]
$$

where $C_{j h}^{(n l)}=\left(x_{j-h+1}\right)^{-\alpha}-\left(x_{j-h}\right)^{-\alpha}$ and with the diagonal terms $C_{j j}^{(n l)}=-\sum_{h \neq j}^{N+1} C_{h=1}^{(n l)}$.

The initial condition of the considered boundary value problem is a linear temperature distribution among prescribed temperature values at the borders $T\left(-\frac{L}{2}, t\right)=T_{1}$ and $T\left(\frac{L}{2}, t\right)=T_{2} \forall t \geqslant 0$. The solution of the temperature equation has been reported in Fig. 5(a)-(d) for cases of $\alpha$ variable and fixed $\tau$ and cases of $\tau$ variable and fixed $\alpha$ in (Fig. 6(a)-(d)) respectively. The inspection of both set of figures shows that the presence of a time lag $\tau$ in the propagation of the initial disturbance corresponds to an evolution of the perturbation front, starting from the borders at $x=-L / 2$ and $x=L / 2$ toward the center of the conductor. The speed of propagation of the thermal wave increases if $\tau$ decreases as it may be captured from direct observation of Fig. 6(a)-(d). Indeed it is shown that the initial distribution is maintained up to the arrival of the temperature waves that yield high temperature gradients at the borders.

\section{Conclusions}

Thermal energy transfer in rigid heat conductors at nano-scales and/or for high frequency processes has been recently modeled by suitable extension of Fourier transport equation in terms of real-order (fractional) derivatives. Such extension relies on the long-tail properties of power-law kernels to describe the slow spatial and temporal decay of temperatures observed at the mesoscale of complex heterogeneous materials. Indeed at these scales, the presence of the material structure influences the thermal energy transport and the use of non-local thermodynamics has became very common to model the scale effect as additional contributions to the transport equations.

In the paper a numerical investigation of the effects induced by non-homogeneous boundary conditions on a recent model of non-local thermodynamics has been reported for diffusive and ballistic heat fluxes. The used model assumes that the thermal energy transfer is due to the superposition of two contributions at the considered observation scale: (i) A phonon collision/ballistic model of thermal energy transfer that is described by means of Fourier/Cattaneo transport equation and (ii) 
a phononic small-scale heat transport accounting for the long-range thermal energy transfer proportional to the relative temperature among interacting locations, to the product of interacting masses and to a proper, material-type decaying function. Restrictions on the functional class of the distance-decaying function have been reported in the paper showing that any decaying function that is strictly positive in the whole conductors' domain is eligible in terms of the Clausius-Planck inequality.

As we assume that the decaying function belongs to the functional class of power-laws of the interacting distances then a fractional-order heat equation with Marchaud-type fractional derivatives of order $\alpha \epsilon[0,1]$ is obtained in unbounded domains. A different scenario appears as bounded conductors are considered since only integral terms of Marchaud fractional operator are retained in the model. This aspect is a peculiarity of the proposed long-range thermal energy transfer that prevent for the ill-conditioning of non-homogeneous Dirichlet and Neumann boundary conditions always encountered in integral non-local approaches.

It is shown that the proposed model yields the phonon-phonon scattering of thermal energy waves outside the thermostatted regions where the changes of the electron density modifies the material conduction parameters yielding a non-uniform temperature field. The model may be extended to coupled thermoelastic problems to highlight the effects of the nonhomogeneous temperature distribution on the stress and strain field observed in materials at the nanoscale.

\section{Acknowledgments}

The author is very grateful to the financial support provided by the PRIN2010-11 "Stability, Control and Reliability of Flexible Structures" with National Coordinator Prof. A. Luongo. Stimulating discussions with Prof. Mario Di Paola are also gratefully acknowledged.

\section{Appendix A. Remarks on fractional calculus}

In this appendix the essential features of fractional calculus will be shortly discussed.

Let us consider a real-valued, Lebesgue integrable function $f(x), x \in \mathbb{R}$ such that $f(x) \in L^{1}$. The left and right RiemannLiouville (RL) fractional-order integrals are defined as:

$$
\begin{aligned}
& \left(I_{+}^{\alpha} f\right)(x)=\frac{1}{\Gamma(\alpha)} \int_{-\infty}^{x} \frac{f(y)}{(x-y)^{1-\alpha}} d y \\
& \left(I_{-}^{\alpha} f\right)(x)=\frac{1}{\Gamma(\alpha)} \int_{x}^{\infty} \frac{f(y)}{(y-x)^{1-\alpha}} d y
\end{aligned}
$$

with $\alpha \in[0,1]$ and $\Gamma(\bullet)$ is the Euler-Gamma function. The left and right fractional derivatives are defined as:

$$
\begin{aligned}
& \left(D_{+}^{\alpha} f\right)(x)=\frac{1}{\Gamma(1-\alpha)} \frac{d}{d x} \int_{-\infty}^{x} \frac{f(y)}{(x-y)^{1-\alpha}} d y \\
& \left(D_{-}^{\alpha} f\right)(x)=\frac{1}{\Gamma(1-\alpha)} \frac{d}{d x} \int_{x}^{\infty} \frac{f(y)}{(y-x)^{1-\alpha}} d y
\end{aligned}
$$

As we assume that function $f(x) \in C^{1}$ with $C^{1}$ the class of continuous functions with continuous first derivative, then the left and right RL fractional derivatives coalesces with the Marchaud (M) fractional operator that is defined as:

$$
\left(\mathbf{D}_{+}^{\alpha} f\right)(x)=\frac{\alpha}{\Gamma(1-\alpha)} \int_{-\infty}^{x} \frac{f(x)-f(y)}{(x-y)^{1+\alpha}} d y=\left(D_{+}^{\alpha} f\right)(x)
$$

for the left $M$ fractional derivative, whereas, the right $M$ fractional derivative is related to the right RL fractional derivative as:

$$
\left(\mathbf{D}_{-}^{\alpha} f\right)(x)=\frac{\alpha}{\Gamma(1-\alpha)} \int_{x}^{\infty} \frac{f(x)-f(y)}{(y-x)^{1+\alpha}} d y=\left(D_{-}^{\alpha} f\right)(x)
$$

The definition of RL and M fractional derivatives operating on functions defined on bounded intervals $[a, b] \subset \mathbb{R}$ involves integral terms as well as algebraic contributions as:

$$
\begin{aligned}
& \left(D_{a^{+}}^{\alpha} f\right)(x)=\frac{f(a)}{\Gamma(1-\alpha)(x-a)^{\alpha}}+\frac{1}{\Gamma(1-\alpha)} \int_{a}^{x} \frac{f(y)^{\prime}}{(x-y)^{\alpha}} d \xi \\
& \left(D_{b^{-}}^{\alpha} f\right)(x)=\frac{f(b)}{\Gamma(1-\alpha)(b-x)^{\alpha}}-\frac{1}{\Gamma(1-\alpha)} \int_{x}^{b} \frac{f(y)^{\prime}}{(y-x)^{\alpha}} d \xi
\end{aligned}
$$

where $f(y)^{\prime}=\frac{d f}{d y}$, showing divergence at the boundaries of the considered domains, unless function $f(x) \rightarrow 0$ faster than $x^{\alpha}$ as $x \rightarrow 0$. Similar considerations hold true also for the $M$ fractional operators defined on bounded support, yielding: 


$$
\begin{aligned}
& \left(\mathbf{D}_{a^{+}}^{\alpha} f\right)(x)=\frac{\alpha f(x)}{\Gamma(1-\alpha)(x-a)^{\alpha}}+\left(\hat{\mathbf{D}}_{a^{+}}^{\alpha} f\right)(x) \\
& \left(\mathbf{D}_{b^{-}}^{\alpha} f\right)(x)=\frac{\alpha f(x)}{\Gamma(1-\alpha)(b-x)^{\alpha}}-\left(\hat{\mathbf{D}}_{b^{-}}^{\alpha} f\right)(x)
\end{aligned}
$$

where $\left(\hat{\mathbf{D}}_{a^{+}}^{\alpha} f\right)(x)$ and $\left(\hat{\mathbf{D}}_{b^{-}}^{\alpha} f\right)(x)$ are, the integral parts of the truncated M fractional operators defined as:

$$
\begin{aligned}
& \left(\hat{\mathbf{D}}_{a^{+}}^{\alpha} f\right)(x)=\frac{\alpha}{\Gamma(1-\alpha)} \int_{a}^{x} \frac{f(x)-f(y)}{(x-y)^{1+\alpha}} d y \\
& \left(\hat{\mathbf{D}}_{b^{-}}^{\alpha} f\right)(x)=\frac{\alpha}{\Gamma(1-\alpha)} \int_{x}^{b} \frac{f(x)-f(y)}{(y-x)^{1+\alpha}} d y
\end{aligned}
$$

Equivalent forms to Eqs. (62) and (63) relative to the M fractional derivatives valid for cases involving $\alpha>1$ may be obtained as we introduce the $l$-order finite differences of function $f(x)$, with $l=\{\alpha\}+1$ and $l>1$ (see e.g. [48]) yielding:

$$
\left(\mathbf{D}_{+}^{\alpha} f\right)(x)=-\frac{1}{\Gamma(-\alpha) A_{l}(\alpha)} \int_{0}^{\infty} \frac{\left(\Delta_{ \pm \xi}^{l} f\right)(x)}{y^{1+\alpha}} d y=\frac{1}{\chi(l, \alpha)} \int_{0}^{\infty} \frac{\left(\Delta_{ \pm \xi}^{l} f\right)(x)}{y^{1+\alpha}} d y
$$

where we denoted $\{\alpha\}$ the integer part of the real number $\alpha$ and the normalization coefficient $\chi(l, \alpha)=-A_{l}(\alpha) \Gamma(-\alpha)$. The fractional finite difference $\left(\Delta_{ \pm \xi}^{l} f\right)(x)$, that appears in the integral term in Eq. (65) and the normalization factor $A_{l}(\alpha)$ are defined as:

$$
\left(\triangle_{ \pm y}^{l} f\right)(x)=\sum_{k=0}^{l}(-1)^{k}\left(\begin{array}{l}
l \\
k
\end{array}\right) f(x-k y) ; \quad A_{l}(\alpha)=\sum_{k=0}^{l}(-1)^{k-1}\left(\begin{array}{l}
l \\
k
\end{array}\right) k^{\alpha}
$$

with $\alpha \in \mathbb{R}^{+}$. The coefficient $A_{l}(\alpha)$ is identically vanishing for integer values of $\alpha=1,2, \ldots, l-1$ whereas the normalization coefficient $\chi(l, \alpha)$ is unbounded as $\alpha \rightarrow l^{-}$and it is finite as $\alpha \rightarrow l^{+}$.

The definitions of Marchaud fractional derivatives applied to scalar functions of simple scalar variables may be extended to scalar functions of multivariable arguments. This extension became more readable as we introduce the Riesz (R) potential operator of function $f$, dubbed $\left(I^{\alpha} f\right)(x)$ that is defined as:

$$
\left(\mathrm{I}^{\alpha} f\right)(x)=\frac{1}{2 \cos (\alpha \pi / 2) \Gamma(\alpha)} \int_{-\infty}^{+\infty} \frac{f(y)}{\|x-y\|^{1-\alpha}} d y=\frac{\left(I_{+}^{\alpha} f\right)(x)+\left(I_{-}^{\alpha} f\right)(x)}{2 \cos (\alpha \pi / 2) \Gamma(\alpha)}
$$

where $\|\bullet\|$ is the Euclidean distance. The inverse operators, namely the D-Riesz fractional differential operator, describing the inverse operator of the Riesz integral, reads (for $0 \leqslant \alpha \leqslant 1$ ):

$$
\left(\mathrm{I}^{\alpha} f\right)^{-1}(x)=\left(\mathrm{D}^{\alpha} f\right)(x)=v(\alpha) \int_{-\infty}^{+\infty} \frac{f(x-y)-f(x)}{\|y\|^{1+\alpha}} d y=v(\alpha) \Gamma(1-\alpha)\left[\left(\mathbf{D}_{+}^{\alpha} f\right)(x)+\left(\mathbf{D}_{-}^{\alpha} f\right)(x)\right]
$$

where $v(\alpha)=[2 \alpha \cos (\alpha \pi / 2) \Gamma(\alpha)]^{-1}$. Mathematical expression reported in Eq. (68) shows that, with the exception of the coefficient $v(\alpha) \Gamma(1-\alpha)$ the inverse Riesz potential operator coincide with the sum of left and right Marchaud fractional derivatives. A different, but equivalent, form of the Riesz fractional operator in Eq. (68) may be written as we introduce the fractional difference operator of order $l$, holding for $\alpha \geqslant 0$ as:

$$
\left(\mathrm{I}^{\alpha} f\right)^{-1}(x)=\left(\mathrm{D}^{\alpha} f\right)(x)=\frac{v(\alpha) \Gamma(1-\alpha)}{\alpha A_{l}(\alpha) \Gamma(-\alpha)} \int_{0}^{\infty} \frac{\left(\Delta_{+y}^{l} f\right)(x)+\left(\Delta_{-y}^{l} f\right)(x)}{y^{1+\alpha}} d y
$$

The expression in Eq. (69) may be easily generalized to the case of fractional generalization of multivariable functions $f(\mathbf{x})$, with $\mathbf{x} \in \mathbb{R}^{n}$ as the $n$-fold integral:

$$
\left(I^{\alpha} f\right)(\mathbf{x})=\frac{1}{\gamma_{n}(\alpha)} \int_{\mathbb{R}^{n}} \frac{f(\mathbf{y})}{\|\mathbf{x}-\mathbf{y}\|^{n-\alpha}} d \mathbf{y} ; \quad \alpha \neq n, n+2, \ldots
$$

with the normalization constant $\gamma_{n}(\alpha)$ is defined in [28] (Eqs. (25) and (26)).

The inverse operator $\left(\mathrm{I}^{\alpha} f\right)^{-1}(\mathbf{x})=\left(\mathrm{D}^{\alpha} f\right)(\mathbf{x})$, termed as the multivariable Riesz fractional differential operator is provided as the $n$-fold integral:

$$
\left(\mathrm{I}^{\alpha} f\right)^{-1}(\mathbf{x})=\left(\mathrm{D}^{\alpha} f\right)(\mathbf{x})=\frac{1}{d_{n, l}(\alpha)} \int_{\mathbb{R}^{n}} \frac{\left(\triangle_{\mathbf{y}}^{l} f\right)(\mathbf{x})}{\|\mathbf{y}\|^{n+\alpha}} d \xi
$$

with $\left(\triangle_{\mathbf{y}}^{l} f\right)(\mathbf{x})$ the centered finite difference that represents the extension to higher-dimensional spaces of Eq. (69) defined as: 


$$
\left(\Delta_{\mathbf{y}}^{l} f\right)(\mathbf{x})=\sum_{k=0}^{l}(-1)^{k}\left(\begin{array}{l}
l \\
j
\end{array}\right) f(\mathbf{x}-k \mathbf{y})
$$

and where $d_{n, l}(\alpha)$ is a proper normalization constant, that involves an explicit dependence of the fractional order $\alpha$ that reads:

$$
d_{n, l}(\alpha)=\beta_{n}(\alpha) \frac{A_{l}(\alpha)}{\sin (\alpha \pi / 2)} ; \quad \beta_{n}(\alpha)=\frac{\pi^{1+n / 2}}{2^{\alpha} \Gamma(1+\alpha / 2) \Gamma(n+\alpha / 2)}
$$

As it has been shown for the 1D case, the Riesz fractional differential operator $\left(\mathrm{D}^{\alpha} f\right)(\mathbf{x})$ may be expressed as the sum of the left and right fractional operators involving fractional differences and henceforth, as in Eq. (69), it may be expressed in terms of the Marchaud fractional derivatives in half-spaces, defined as:

$$
\left(\mathrm{D}^{\alpha} f\right)(\mathbf{x})=\frac{\chi_{\bar{l}}(\bar{\alpha})}{d_{n, l}(\bar{\alpha})} \frac{1}{\chi_{\bar{l}}(\bar{\alpha})}\left[\int_{\mathbb{R}_{+}^{n}} \frac{\left(\Delta_{+\mathbf{y}}^{\bar{l}} f\right)(\mathbf{x})}{\mathbf{y}^{1+\bar{\alpha}}} d \mathbf{y}+\int_{\mathbb{R}_{-}^{n}} \frac{\left(\Delta_{-\mathbf{y}}^{\bar{l}} f\right)(\mathbf{x})}{(-\mathbf{y})^{1+\bar{\alpha}}} d \mathbf{y}\right]=\frac{\chi_{\bar{l}}(\bar{\alpha})}{d_{n, l}(\bar{\alpha})}\left[\left(\mathbf{D}_{+}^{\alpha} f\right)(\mathbf{x})+\left(\mathbf{D}_{-}^{\alpha} f\right)(\mathbf{x})\right]
$$

with $\bar{\alpha}=(n-1)+\alpha$ and $\bar{l}=(n-1)+l=\bar{\alpha}$. Eq. (74) may also be written, under the assumption $0 \leqslant \alpha \leqslant 1$ as (see [55, Eqs. (25) and (26)]):

$$
\left(D^{\alpha} f\right)(\mathbf{x})=\frac{1}{d_{n, l}(\bar{\alpha})} \int_{\mathbb{R}^{n}} \frac{f(\mathbf{y})-f(\mathbf{x})}{\|\mathbf{x}-\mathbf{y}\|^{n+\alpha}} d \xi=\frac{\chi_{\bar{l}}(\bar{\alpha})}{d_{n, l}(\bar{\alpha})}\left[\left(\mathbf{D}_{+}^{\alpha} f\right)(\mathbf{x})+\left(\mathbf{D}_{-}^{\alpha} f\right)(\mathbf{x})\right]
$$

that corresponds, in case of multivariable function fields $f(\mathbf{x})$, to a relation between the Riesz and the Marchaud differential operators analogous to that involving scalar variable functions reported in Eq. (69).

\section{References}

[1] Jolley K, Gill S. Modelling transient heat conduction in solids at multiple length and time scales: a coupled non-equilibrium molecular dynamics/ continuum approach. J Comput Phys Sci 2009;228:7412.

[2] Cahill D, Ford W, Goodson K, Mahan G, Majumdar A, Maris H, Merlin R, Philpot S. Nanoscale thermal transport. J Appl Phys 2003;93:793.

[3] Jolley K, Gills SPA. Modeling transient heat conduction at multiple length and time scale: a coupled equilibrium molecular dynamics/continuum approach. In: Pyrz R, Rauhe JC, editors. Proc IUTAM symp nanomat nanosyst. London: Pergamon Press; 2008.

[4] Caputo MG. Vibrations of a thin viscoelastic layer with a dissipative memory. J Acoust Soc Am 1974;56:793-904.

[5] Sherief HH, El-Sayed A, Abd-El-Latief A. Fractional order theory of thermoelasticity. Int J Solids Struct 2010;47:269-75.

[6] Youssef HM. Theory of fractional-order generalized thermoelasticity. J Heat Transfer 2010;47:269-75.

[7] Povstenko YZ. Fractional heat conduction equations and associated thermal stress. J Therm Stress 2005;28:83-102.

[8] Povstenko YZ. Two-dimensional axisymmetric stresses exerted by instantaneous pulses and sources of diffusion in an infinite space in case of timefractional diffusion equation. Int J Solids Struct 2007;44:2324-48.

[9] Povstenko YZ. Theory of thermoelasticity based on space-time fractional heat conduction equation. Phys Scr 2009;T136:014017.

[10] Podlubny I. Fractional differential equations. New-York: Accademic Press; 1998.

[11] Mainardi F, Luchko Y, Pagnini G. The fundamental solution of the space-time fractional diffusion equation. Frac Calc Appl Anal 2001;4:153-92.

[12] Tarasov VE, Zaslavsky GM. Conservation laws and hamiltonian's equations for systems with long-range interaction and memory. Commun Nonlinear Sci Numer Simul 2001;13:1870-8.

[13] Tarasov VE. Fractional integro-differential equations for electromagnetic waves in dielectric media. Theor Math Phys 2009;158:153-9.

[14] Zumofen G, Klafter J. Scale invariant motion in intermittent chaotic systems. Phys Rev E 1993;47:851-63.

[15] Metzler R. Generalized Chapman-Kolmogorov equation: an unified approach to the description of anomalous transport in external fluids. Phys Rev E 2000;62:6233-45.

[16] Compte A. Stochastic foundation of fractional dynamics. Phys Rev E 1996;47:4191-3.

[17] Metzler R, Klafter J. The random walk's guide to anomalous diffusion: a fractional dynamics approach. Phys Rep 2000;339:1-77.

[18] Metzler HR, Blumen A, Nonnenmacher TF. Generalized viscoelastic models: their fractional equations with solutions. J Phys A: Math Gen 1995;28:6567-84.

[19] Friedrich C. Mechanical stress relaxation in polymers: fractional integral model versus fractional differential model. J Nonnewtonian Fluid Mech 1993;46:307-14.

[20] Metzler HR, Nonnenmacher TF. Fractional relaxation processes and fractional rheological models for the description of a class of viscoelastic materials. Int J Plast 2003;19:941-59.

[21] Paola MD, Zingales M. Exact mechanical models of fractional hereditary materials (fhm). J Rheol 2012;58:986-1004.

[22] Paola MD, Pinnola F, Zingales M. A discrete mechanical model of fractional hereditary materials (fhm). Meccanica 2013;48:1573-86.

[23] Craiem D, Armentano R. A fractional derivative model to describe arterial viscoelasticity. Biorheol 2013;44:251-63.

[24] Paola MD, Pinnola F, Zingales M. Fractional differential equations and related exact mechanical models. Comput Math Appl 2013;66:608-20.

[25] Deseri L, DiPaola M, Zingales M, Pollaci P. Power-law hereditariness of hierarchical fractal bones. Int J Numer Methods Biomed Eng 2013;29:1338-60.

[26] Deseri L, Zingales M, Pollaci P. The state of fractional hereditary materials (fhm). Dis Cont Dyn Syst; in press.

[27] Deseri L, Golden MJ, Fabrizio M. The concept of a minimal state in viscoelasticity: new free energies and applications to pdes. Arch Ration Mech Anal 2006; 181:43-96.

[28] Piero GD, Deseri L. On the concepts of state and free energy in linear viscoelasticity. Arch Ration Mech Anal 1997;138:1-35.

[29] Deseri L, Golden J. The minimum free energy for continuous spectrum materials. SIAM J Appl Math 2007;67:869-92.

[30] Deseri L, Marcari G, Zurlo G. Thermodynamics, continuum mechanics. In: Merodio, editor. EOLSS-UNESCO encyclopedia saccomandi [Chap. 5].

[31] Lazopoulos K. Non-local continuum mechanics and fractional calculus. Mech Res Commun 2006;33:753-7.

[32] Cottone G, Paola M, Zingales M. Fractional mechanical model of non-local continuum. Lect Note Electr Eng 2009;11:95-103.

[33] DiPaola M, Zingales M. Long-range cohesive interactions of non-local continuum faced by fractional calculus. Int J Solids Struct 2008;45:5642-59.

[34] DiPaola M, Zingales M. Fractional differential calculus for 3d mechanically-based non-local elasticity. Int J Multi Comput Eng 2011;9:579-97.

[35] Failla G, Santini A, Zingales M. Solution strategies for 1d elastic continuum with long-range interactions: smooth and fractional decay. Mech Res Commun 2010;37:13-21. 
[36] Carpinteri A, Cornetti P, Sapora G, DiPaola M, Zingales M. Fractional calculus in solid mechanics: local versus non-local approach. Phys Scr 2009;T136:014003.

[37] Cottone G, Paola M, Zingales M. Elastic waves propagation in 1d fractional non-local continuum. Phys E: Low-Dim Syst Nanostruct 2009;42:95-103.

[38] Zingales M. Wave propagation in 1d elastic solids in presence of long-range central interactions. J Sound Vib 2011;330:3973-89.

[39] Paola M, Failla G, Pirrotta A, Sofi A, Zingales M. The mechanically based non-local elasticity: an overview of main results and future challenges. Philos Trans R Soc Lond A 2013;371 [art no. 20120433].

[40] Eringen C. An unified theory of thermomechanical materials. Int J Eng Sci 1966;4:179-202.

[41] Eringen C. Theory of nonlocal thermoelasticity. Int J Solids Struct 1974;12:1063-77.

[42] Eringen CA. A non-local linear elasticity theory. Int J Eng Sci 1972;4:179-202.

[43] Ardito R, Comi C. Nonlocal thermoelastic damping in microelectromechanical resonators. J Eng Mech 2009;135:214-20.

[44] Zingales M, Elishakoff I. Localization of the bending response in presence of axial load. Int J Solids Struct 2000;37:6739-53.

[45] Jou D, Lebon G, Mongioví M, Peruzza RA. Entropy flux in non-equilibrium thermodynamics. Phys A: Math Theor 2004;338:445-57.

[46] Mongioví MS. On linear extended thermodynamics of a non-viscous fluid in presence of heat flux. J Nonequilibr Therm 2000;25:31-47.

[47] Straughan B. Heat waves: applied mathematical sciences, vol. 177. New York: Springer; 2011.

[48] Cattaneo C. Sulla conduzione del calore, Atti del Seminario di Mat. Fis. UniversitÃ di Modena; 3, 1947 [in Italian].

[49] Metzler R, Nonnematcher TF. Fractional diffusion, waiting time distribution and Cattaneo-type equation. Phys Rev E 1998;47:6409-14.

[50] Compte A, Metzler R. The generalized Cattaneo equation for the description of anomalous transport process. J. Phys. A 1997;47:7277-82.

[51] Lebon G, Jou D, Casas-Vàzquez J. Extended irreversible thermodynamics. New York: Springer; 2005.

[52] Jou D, Casas-Vàzquez J, Lebon G. Understanding non-equilibrium thermodynamics. New York: Springer; 2010.

[53] Borino G, DiPaola M, Zingales M. A non-local model of fractional heat conduction in rigid bodies. E Phys J: S-T 2010;193:173-84.

[54] Mongioví M, Zingales M. A non-local model of thermal energy transport: the fractional temperature equation. J Heat Mass Transfer 2013;67:593-601.

[55] Samko S, Kilbas A, Marichev O. Fractional integrals and derivatives. Amsterdam: Gordon Breach; 1989. 